\section{Abstract}

\title{
Uncertainty estimation and prediction for interdisciplinary ocean dynamics
}

\author{
Pierre F.J. Lermusiaux \\ Harvard University, Division of Engineering and Applied Sciences, Pierce Hall G2A, 29 Oxford Street, Cambridge, MA 02318, USA
}

Received 6 September 2005; received in revised form 25 January 2006; accepted 9 February 2006

Scientific computations for the quantification, estimation and prediction of uncertainties for ocean dynamics are developed and exemplified. Primary characteristics of ocean data, models and uncertainties are reviewed and quantitative data assimilation concepts defined. Challenges involved in realistic data-driven simulations of uncertainties for four-dimensional interdisciplinary ocean processes are emphasized. Equations governing uncertainties in the Bayesian probabilistic sense are summarized. Stochastic forcing formulations are introduced and a new stochastic-deterministic ocean model is presented. A computational methodology and numerical system, Error Subspace Statistical Estimation, for the efficient estimation and prediction of oceanic uncertainties based on these equations is then outlined. Capabilities of the ESSE system are illustrated in three data-assimilative applications: estimation of uncertainties for physical-biogeochemical fields, transfers of ocean physics uncertainties to acoustics, and real-time stochastic ensemble predictions with assimilation of a wide range of data types. Relationships with other modern uncertainty quantification schemes and promising research directions are discussed. (c) 2006 Elsevier Inc. All rights reserved.

Keywords: Ocean modeling; Physical-biological-acoustical interactions; Multiscale; Atmospheric and weather forecasting; Stochastic processes; Stochastic ocean models; Data assimilation; Estimation theory; Uncertainty analysis

\section{Introduction}

The ocean physics involves a multitude of phenomena occurring on multiple scales, from molecular and turbulent processes to decadal variations and climate dynamics. Life takes place in the ocean, from bacteria and plankton cells to fish and mammals. Marine ecosystems also involve multiple phenomena, for example consider plankton blooms, biomass cycles or regime shifts in fish populations. Oceanic processes cover a wide range of space scales, from about $1 \mathrm{~mm}$ to $10,000 \mathrm{~km}$, and of time scales, from about $1 \mathrm{~s}$ to 100 years and more. Features and properties in the ocean interact over these scales and significant interactions occur predominantly over certain ranges of scales, which are usually referred to as scale windows. For example, the

E-mail address: pierrel@pacific.harvard.edu.

URL: http://www.deas.harvard.edu/ pierrel. 
internal weather of the sea, the so-called oceanic mesoscale, mainly consists of phenomena occurring over a day to months and over kilometers to hundreds of kilometers. This is one of the most energetic scale windows in the ocean and the present methodological and computational study on the quantification and large-scale simulation of uncertainties focuses on this window of oceanic processes.

Oceanic physical features, such as currents, fronts, eddies, tides, internal waves, solitons and turbulence influence the distribution of organisms, the rates of biological activity and the performance of underwater acoustic remote sensing. Many physical, biological and chemical features and processes interact. For example, consider oceanic primary productivity, the oceanic food web or the biological pump and its role in changing the global carbon cycle. Several important oceanic processes are interdisciplinary, multiscale and nonlinear [69], and, in coastal oceans, strong episodic and patchy events contribute to these dynamics [62]. Often, this variability can be neither sampled nor modeled on a sustained and substantial basis, at scales and accuracies sufficient for a definite representation. Marine data are required for realistic studies but are limited in coverage in time and space. Comprehensive ocean models are approximate because of practical simplifications, inexact representations or parameterizations, and numerical implementations. This results in uncertainties or differences between the actual values (unknown) and the measured or modeled values of physical, biological and acoustical fields and properties.

A comprehensive prediction should include the reliability of estimated quantities. This allows an adequate use of these estimates in a scientific or operational application. In a prediction with a model integrating either in time and/or in space, errors in the initial data (initial conditions), boundary conditions and models themselves impact accuracy. Predicted uncertainties then contain the integrated effects of the initial error and of the errors introduced continuously during model integration.

Variability and uncertainty are inherently related, and this relation affects the attributions of errors, e.g. [51]. For any estimate, the portion of variability that is expected to contain errors contributes to uncertainty. For example, variability that is totally unresolved is pure uncertainty. Mathematically, uncertainty can be defined here by the probability density function (PDF) of the error in the estimate. Uncertainties are thus assumed measurable. Error refers to the difference between the truth and the estimate. Uncertainties are often represented by low order characteristics or integrals of the error PDF, e.g., the moments or confidence intervals. For example, for the classic standard deviation, the representation is the square root of the mean squared error. Since ocean fields are four-dimensional, straightforward uncertainty representations are here also fields, with structures in time and space.

Realistic simulations of four-dimensional ocean fields are carried out over broad numerical domains, e.g. $\mathrm{O}(10-1000) \mathrm{km}$ for $\mathrm{O}(10-1000)$ days. The number of grid points and thus of discretized state variables are very large, usually of $\mathrm{O}\left(10^{5}-10^{7}\right)$. On the other hand, ocean data are limited in temporal and spatial coverage. Commonly, the number of data points for an at-sea sampling campaign is of $\mathrm{O}\left(10^{4}-10^{5}\right)$. For substantial scientific advances and to reduce uncertainties, the sources of information, the various data and dynamical models, are combined by data assimilation [68,71]. This combination is challenging and expensive to carry out, but optimal in the sense that each type of information is weighted in accord with its uncertainty. In principle, this process provides better estimates of parameters and properties than can be obtained by using only the observations or models alone. In coupled data assimilation, multivariate correlations are used. For example, physical data improve biological fields and biological data improve physical fields. Of course, should optimal field and error estimates fail to be accurate, a priori assumptions about uncertainties are revised, and models and data sets improved. Once oceanic properties have been estimated, specific dynamical events can be studied and corresponding simpler models can be derived, leading to better understanding.

In what follows, we review primary characteristics of ocean data, models and uncertainties, and define concepts linked to data assimilation (Section 2). Fundamental equations including novel oceanic stochastic forcing formulations and a computational methodology and numerical system for the estimation and prediction of realistic ocean uncertainties are then presented (Section 3). The system, error subspace statistical estimation, has been developed and successfully utilized for real-time data assimilation and uncertainty forecasting. It is illustrated based on three new interdisciplinary data-assimilative uncertainty applications: physical-biogeochemical estimations in Massachusetts Bay, physics-to-acoustics transfers in the Middle Atlantic Bight and sustained physical predictions in the Monterey Bay region (Section 4). Relationships with other modern uncer- 
84 tainty quantification schemes and promising directions are then discussed (Section 5). After the conclusions (Section 6), details on the new stochastic-deterministic coupled models are given (Appendix A).

\section{2. Interdisciplinary data, models and data assimilation}

The platforms that can be utilized today to measure oceanic properties in situ consist mostly of ships, aircraft, moored/fixed buoys, drifters and underwater vehicles including gliders [14]. Remotely sensed observations are also available, for example from satellites or coastal radar, which provide repeated larger-scale snapshots of surface properties of oceanic regions. Platforms at sea can be equipped with several sensors measuring physical properties of the ocean, such as currents, temperature, salinity or pressure. The resulting data are point-wise observations along the path of the sensor, for example successive temperature vertical profiles. Biological and chemical properties are also often measured in situ and common data types include available light radiation, fluorescence, dissolved nitrogen concentrations, or plankton species and concentrations. Underwater sound propagation is influenced by the ocean's physics and biology, in a large part through variations in the sound speed and scattering processes. Integrated oceanic effects between a source and receiver are thus contained in most acoustic observations. For example, acoustic propagation data can be used to estimate current velocities and water densities by direct inversion. In addition, the backscatter from acoustic propagation contains useful information on the abundance and biology of organisms that are responsible for the backscatter. Biological inference from acoustic observation is emerging [29,60]. Even though oceanic measurements are the source of ground truth for scientific studies, sensors require calibrations. Raw measurements are processed and often de-aliased, filtered or averaged. Each ocean datum is thus associated with some uncertainties.

Most physical ocean models are derived from the Navier-Stokes equations in a rotating frame of reference. Practical assumptions are used to limit the range of modeled scales. A Boussinesq fluid, or small variations of density about a state of reference, is usually assumed. The turbulent flow is commonly reduced to a scale window of interest (here mostly the mesoscale) by averaging. Turbulent parameterizations/closures are then utilized. Finally, the thinness approximation and assumption of hydrostatic balance can be made (vertical scales much smaller than horizontal scales). The result of these simplifications is the so-called primitive equations (PE) of physical ocean dynamics [66], which are employed here (Appendix A.1). Acoustic models are also derived from Navier-Stokes and are usually based on a wave equation for the sound pressure [43]. Even though much progress has been made in marine ecosystem modeling, e.g. [45,34], equations as fundamental as Navier-Stokes are not yet available. For lower trophic levels, most models are based on advection-reaction-diffusion equations: they differ in their structure, the number of state variables employed and the parameterizations utilized. All of the above simplifications, approximate representations and parameterizations employed for four-dimensional ocean modeling lead to uncertainties. In addition, numerical algorithms discretize the approximate models for numerical simulations. Most numerical ocean models are based on finite differences, but some finite element models are also employed. Computations are subject to errors associated with the algorithms used and the limitations of the computer. To represent all of these model uncertainties or at least their dominant components, stochastic error models are starting to be employed, e.g., for representing the statistical effects of sub-mesoscales and internal tidal phenomena in mesoscale resolution models (see Section 3.1.1 and Appendix A.2).

Today, real-time ocean predictions for scientific and operational applications require a systemic approach that synthesizes theory, data and numerical computations [69]. The concept of ocean observing and prediction systems for field and parameter estimations has recently been defined with three major components: (i) an observational network: a suite of platforms and sensors for specific tasks, including data management and analysis schemes; (ii) a suite of interdisciplinary dynamical models; and, (iii) importantly, data assimilation schemes and scientific analyses. Ideal systems are modular, based on distributed information systems providing sharable, scalable, flexible and efficient workflows [65]. Such systems are starting to be utilized on global, basin and regional scales [67], with varied levels of complexity and accuracy.

For melding measurements with dynamical models via data assimilation (DA), measurement models that link the dynamical model variables and parameters to the observations are needed. These measurement mod- 
els include uncertainties because the sensors, data collection procedures and relationships among data and dynamical variables, are approximate. The data and measurement models, and the dynamical models, are combined in accord with their prior uncertainty estimates using a DA criterion. This criterion determines how each source of information is weighted. The results of the DA are melded estimates of the ocean state, parameters and/or model structures themselves. These posterior estimates correspond to field estimation, parameter estimation and model estimation, respectively. It is the multivariate relations among model variables or observations that form the basis for multivariate DA: i.e., the correction of a property from the measurement of another. The generic DA problem is summarized as:

Dyn. models: $\mathrm{d} \phi_{i}+\mathbf{u} \cdot \nabla \phi_{i} \mathrm{~d} t-\nabla\left(K_{i} \nabla \phi_{i}\right) \mathrm{d} t=\mathscr{B}_{i}\left(\phi_{1}, \ldots, \phi_{i}, \ldots, \phi_{n}\right) \mathrm{d} t+\mathrm{d} \eta_{i}$

Param. eqns.: $\mathrm{d} P_{\ell}=\mathscr{C}_{\ell}\left(\phi_{1}, \ldots, \phi_{i}, \ldots, \phi_{n} ; P_{\ell}\right) \mathrm{d} t+\mathrm{d} \zeta_{\ell}$

Meas. models: $y_{j}=\mathscr{H}_{j}\left(\phi_{1}, \ldots, \phi_{i}, \ldots, \phi_{n} ; P_{\ell}\right)+\epsilon_{j}$

DA criterion: $\min _{\phi_{i}, P_{\ell}} J\left(\mathrm{~d} \eta_{i}, \mathrm{~d} \zeta_{\ell}, \epsilon_{j}, q_{\eta}, q_{\zeta}, q_{\epsilon}\right)$

To obtain Eq. (1a), the deterministic partial differential equations (PDEs), see Appendix A.1, for $\phi_{i}=u, v, T, \ldots, Z, \ldots, p_{s}$ with $i=(1, \ldots, n)$ are modified and manipulated to PDEs with stochastic forcings $\mathrm{d} \eta_{i}$ so as to represent model uncertainties. The rationale chosen and governing equations for the stochastic forcings are in (Section 3). Model parameters (diffusivities, biological rates, etc.), $P_{\ell}=\left\{K_{i}, R_{i}, \ldots\right\}$ with $\ell=(1, \ldots, p)$, are also represented by an equation (Eq. (1b)) with stochastic forcings $\mathrm{d} \zeta_{\ell}$, where $\mathscr{C}_{\ell}$ are functionals that describe the deterministic evolution of the parameters with time and space. The resulting Eqs. (1a) and (1b) are here written as Itô stochastic PDEs, e.g. [37,25]. The state variables $\phi_{i}$ are related to observations $y_{j}$ (temperature, sea surface height, transmission loss, coastal ocean dynamics application radar data, fluorescence, etc., with $j=1, \ldots, m)$ via measurement operators $\mathscr{H}_{j}$ and with stochastic forcings $\epsilon_{j}$ (Eq. (1c)). The functionals $\mathscr{B}_{i}, \mathscr{C}_{\ell}$ and $\mathscr{H}_{j}$ can depend on the values of parameters $P_{\ell}$ : e.g., $\mathscr{C}_{\ell}$ is in general a function of $P_{\ell}$ and parameters can be directly measured in $\mathscr{H}_{j}$. The terms $\mathrm{d} \zeta_{\ell}$ in Eq. (1b) thus lead in general to multiplicative noise: e.g., $\mathrm{d} \zeta_{\ell}$ 's that multiply functionals of $\phi_{i}$ 's in Eq. (1a). Most parameter estimation problems lead to nonlinear estimations and involve multiplicative noise. The DA or melding criterion (Eq. (1d)) involves in general the minimization of a functional $J$ of the stochastic forcings $\left(\mathrm{d} \eta_{i}, \mathrm{~d} \zeta_{\ell}, \epsilon_{j}\right)$ and their a priori statistical properties here denoted by $\left(q_{\eta}, q_{\zeta}, q_{\epsilon}\right)$, subject to the constraints in Eqs. (1a)-(1c). This optimization leads to the posterior estimates of $\phi_{i}$ and $P_{\ell}: \hat{\phi}_{i}$ and $\hat{P}_{\ell}$.

If models/data are used as strong constraints, the terms $\mathrm{d} \eta_{i}, \mathrm{~d} \zeta_{\ell}$ or $\mathrm{d} \epsilon_{j}$ are null. If they are used as weak constraints, the PDFs of the stochastic forcings are specified and used in Eq. (1d). Importantly, these stochastic forcings do not need to have a zero mean, but Eqs. (1a)-(1c) can always be written so that they do, by transferring the means to the deterministic terms, $\mathscr{B}_{i}, \mathscr{C}_{\ell}$ and $\mathscr{H}_{j}$. Data-model misfits/residuals refer to the differences between the data and the model estimated values of the data, $y_{j}-\mathscr{H}_{j}\left(\hat{\phi}_{1}, \ldots, \hat{\phi}_{i}, \ldots, \hat{\phi}_{n}\right)$.

A number of simple to complex methods have been developed and used for DA in engineering, meteorology and oceanography $[3,80,61,70,40]$. An overview is presented in Section 3 of [68], with an appendix that provides equations for various methods. Most schemes are derived from estimation theory, control theory (for variational or calculus of variations approaches) or optimization theory (for direct methods). Estimation theory schemes solve a forward/filtering problem or a smoothing/inverse problem. Control theory schemes solve a smoothing problem. Almost all schemes are linked to a minimization of an error norm or criterion (Eq. (1d)). Many methods are based on least-squares norms.

The posterior uncertainties and data-model misfits, and their agreements or discrepancies, are useful to evaluate the assimilation scheme. They also provide the inputs to two essential assimilation feedbacks. First, the types and locations of the observations that are most needed can be determined and their characteristics predicted by adaptive sampling. Second, the model properties that need the most improvements can be identified and their characteristics estimated by adaptive modeling [56]. Some DA schemes only require the specification of uncertainties for the inputs in Eqs. (1a)-(1c). They do not require an explicit estimation of uncertainties for the outputs, the model field and parameter estimates, $\hat{\phi}_{i}$ and $\hat{P}_{\ell}$. Presently, the goal is to estimate such uncertainties explicitly and the corresponding methodology is described and illustrated next. Uncertainty estimates enhance the DA scheme and allow for adaptive sampling and adaptive modeling. 


\section{2}

183

184

185

186

187

188

189

190

191

192

193

194

\section{5}

196

197

198

199

200

201

202

203

204

205

289

209

210

211

212

213

214

215

216

217

218

219

220

221

222

223

224

\section{Uncertainty estimation methodology}

DA can reduce uncertainties but there always remain some irreducible errors which need to be represented. One usually first identifies the dominant processes that are not accounted for or not resolved and that are expected to impact uncertainties. These are the inputs, the priors, in a Bayesian framework. Uncertainty predictions can then be carried out using evolution equations for the error PDFs (of the dynamics and parameters). When observations are made, these PDFs are updated by combining them with the new data values and their PDFs, using Bayes' rule. If everything is linear and Gaussian, Bayes' rule reduces to the Kalman filter. Important properties are the: (i) conditional mean which is the minimum error variance estimate; and (ii) error covariances which are simple but essential components of the error statistics. For complex interdisciplinary ocean systems, efficient methodologies for the representation, prediction and reduction of uncertainties using this probabilistic framework is an important research issue. In what follows, we outline some fundamental equations, present the computational method for ocean uncertainty predictions and list some specifics on its implementation.

\subsection{Fundamental equations}

The deterministic component in Eqs. (1a) and (1b) are nonlinear PDEs defined on an infinite dimensional space of functional fields. In the formal sense, one could continue in this setting, e.g. [58], and provide some equations for uncertainty evolutions. For the purpose of computations in finite-dimensional spaces, we assume for ease of notation that spatial dimensions have been discretized in some fashion. The ocean state fields $\phi_{i}$, their parameter fields $P_{\ell}$ and all boundary conditions are thus discretized and concatenated into an interdisciplinary and coupled state vector $\mathbf{x}$, of large but finite dimension. In Eq. (1c), observations are taken at discrete instants, e.g. $t_{k} \geqslant t_{0}$. Their concatenation into a vector defines the data vector $\mathbf{y}_{k}^{o}$ (the notation of [36] is employed).

Using the classic formalism of continuous-discrete estimation [37], the spatially discretized version in Eqs. (1a) and (1b) are combined into a single equation for the augmented state vector $\mathbf{x}$. Dynamics and observations are then described by

$$
\begin{aligned}
& \mathrm{d} \mathbf{x}=\mathscr{M}(\mathbf{x}, t)+\mathrm{d} \boldsymbol{\eta} \\
& \mathbf{y}_{k}^{\mathrm{o}}=\mathscr{H}\left(\mathbf{x}_{k}, t_{k}\right)+\boldsymbol{\epsilon}_{k}
\end{aligned}
$$

where $\mathscr{M}$ and $\mathscr{H}$ are the model and measurement model operator, respectively. The stochastic forcings $\mathrm{d} \boldsymbol{\eta}$ and $\boldsymbol{\epsilon}_{k}$ are Wiener-Brownian motion processes, $\boldsymbol{\eta} \sim \mathscr{N}(0, \mathbf{Q}(t))$, and white Gaussian sequences, $\boldsymbol{\epsilon}_{k} \sim \mathscr{N}\left(0, \mathbf{R}_{k}\right)$, respectively. In other words, $\mathscr{E}\left\{\mathrm{d} \boldsymbol{\eta}(t) \mathrm{d} \boldsymbol{\eta}^{\mathrm{T}}(t)\right\} \doteq \mathbf{Q}(t) \mathrm{d} t$ (Section 3.1.1). As a reminder, a form of multiplicative noise is included in Eq. (2a) since the governing equations for model parameters (Eq. (1b)) contain stochastic forcing. The initial conditions are also uncertain and $\mathbf{x}\left(t_{0}\right)$ is random with a prior $\operatorname{PDF}, p\left(\mathbf{x}\left(t_{0}\right)\right)$, i.e., $\mathbf{x}\left(t_{0}\right)=\widehat{\mathbf{x}}_{0}+\mathbf{n}(0)$ with $\mathbf{n}(0)$ random. Of course, vectors and operators in Eqs. (2a) and (2b) are multivariate which impacts the PDFs: e.g., their moments are also multivariate.

The estimation problem at time $t$ consists of combining all available information on $\mathbf{x}(t)$, the dynamics and measurements (Eqs. (2a) and (2b)), their prior distributions and the initial conditions $p\left(\mathbf{x}\left(t_{0}\right)\right)$. Defining the set of all observations prior to time $t$ by $\mathbf{y}_{t-}$, the conditional PDF of $\mathbf{x}(t), p\left(\mathbf{x}, t \mid \mathbf{y}_{t-}\right)$, contains all of this information and is the solution for the prediction to time $t$. For the filtering problem at $t_{k}$, it is $p\left(\mathbf{x}, t_{k} \mid \mathbf{y}_{0}^{\mathrm{o}}, \ldots, \mathbf{y}_{k}^{\mathrm{o}}\right)$. Under classic hypotheses of differentiability and continuity, e.g. [37,25], $p\left(\mathbf{x}, t \mid \mathbf{y}_{t-}\right)$ is governed between observations by the Fokker-Planck equation or Kolmogorov's forward equation (Eq. (3a)). At measurement times $t_{k}$, one can simply apply Bayes' rule and use the assumed white property of $\epsilon_{k}$ to obtain the update Eq. (3b)

$$
\begin{aligned}
& \frac{\partial p\left(\mathbf{x}, t \mid \mathbf{y}_{t-}\right)}{\partial t}=-\sum_{i=1}^{n} \frac{\partial\left(p\left(\mathbf{x}, t \mid \mathbf{y}_{t-}\right) \mathscr{M}_{i}(\mathbf{x}, t)\right)}{\partial \mathbf{x}_{i}}+\frac{1}{2} \sum_{i, j=1}^{n} \frac{\partial^{2}\left(p\left(\mathbf{x}, t \mid \mathbf{y}_{t-}\right) \mathbf{Q}_{i j}\right)}{\partial \mathbf{x}_{i} \partial \mathbf{x}_{j}} \\
& p\left(\mathbf{x}, t_{k} \mid \mathbf{y}_{0}^{\mathrm{o}}, \ldots, \mathbf{y}_{k}^{\mathrm{o}}\right)=\frac{p\left(\mathbf{y}_{k}^{\mathrm{o}} \mid \mathbf{x}\right) p\left(\mathbf{x}, t_{k} \mid \mathbf{y}_{0}^{\mathrm{o}}, \ldots, \mathbf{y}_{k-1}^{\mathrm{o}}\right)}{\int p\left(\mathbf{y}_{k}^{\mathrm{o}} \mid \chi\right) p\left(\chi, t_{k} \mid \mathbf{y}_{0}^{\mathrm{o}}, \ldots, \mathbf{y}_{k-1}^{\mathrm{o}}\right) \mathrm{d} \chi}
\end{aligned}
$$


228 In Eq. (3a), the last term is equal to $\frac{1}{2} \operatorname{tr}\left(\frac{\partial^{2} p}{\partial \mathbf{x} \partial \mathbf{x}} \mathbf{Q}\right)$. Note that $p\left(\mathbf{x}, t \mid \mathbf{y}_{t-}\right)$ is itself random since it depends on data values prior to $t$. Equations for governing the moments, modes, etc., of the PDF can be obtained from Eqs. (3a) and (3b). When data are assumed to be continuous in time, Eqs. (3a) and (3b) are replaced by the Kushner equation if PDFs are retained or by the Zakai equation if a non-normalized form is employed $[82,37,46]$. Both explicitly depend on data value increments.

Because of the oceanic challenges (Sections 1 and 2), estimating the full $p\left(\mathbf{x}, t \mid \mathbf{y}_{t-}\right)$ for realistic applications is challenging. For efficient sub-optimal estimates, we focus on the conditional mean and error covariance matrix $\mathbf{P}=\mathscr{E}\left\{(\mathbf{x}-\widehat{\mathbf{x}})(\mathbf{x}-\widehat{\mathbf{x}})^{\mathrm{T}}\right\}$, of initial conditions $\mathbf{P}(0)$. Using the Itô rule (e.g. [37,25]) and Eq. (3a), the evolution of $\mathbf{P}$ in between observations is governed by Eq. (4a), where $\langle\cdot\rangle$ denotes expectations. The update of $\mathbf{P}$ at data times $t_{k}$ is derived from Eq. (3b) and given by Eq. (4b), where $\langle\cdot\rangle_{-}$are expectations over $p\left(\mathbf{x}, t \mid \mathbf{y}_{t-}\right)$.

$$
\begin{aligned}
& \frac{\mathrm{d} \mathbf{P}}{\mathrm{d} t}=\left\langle(\mathbf{x}-\widehat{\mathbf{x}})(\mathscr{M}(\mathbf{x})-\mathscr{M}(\widehat{\mathbf{x}}))^{\mathrm{T}}\right\rangle+\left\langle(\mathscr{M}(\mathbf{x})-\mathscr{M}(\widehat{\mathbf{x}}))(\mathbf{x}-\widehat{\mathbf{x}})^{\mathrm{T}}\right\rangle+\mathbf{Q} \\
& \mathbf{P}_{k}(+)=\frac{\left\langle\mathbf{x}_{k} \mathbf{x}_{k}^{\mathrm{T}} p\left(\mathbf{y}_{k}^{\mathrm{o}} \mid \mathbf{x}_{k}\right)\right\rangle_{-}}{\left\langle p\left(\mathbf{y}_{k}^{\mathrm{o}} \mid \mathbf{x}_{k}\right)\right\rangle_{-}}-\widehat{\mathbf{x}}_{k}(+) \widehat{\mathbf{x}}_{k}(+)^{\mathrm{T}}
\end{aligned}
$$

The evolution of $\mathbf{P}$ depends on all moments and on data values prior to $t$. It is only for linear systems that the covariance evolution does not depend on data values (e.g. $[37,44,10]$ for related studies). For minimum error variance, the goal is to minimize the trace of the a posteriori error covariance $\mathbf{P}_{k}(+)$ : i.e., find $\mathbf{x}_{k}$ such that $J_{k}=\operatorname{tr}\left[\mathbf{P}_{k}(+)\right]$ is minimized using the data up to time $t_{k} / t_{N},\left[\mathbf{y}_{0}, \ldots, \mathbf{y}_{k} / \mathbf{y}_{N}\right]$, for the filtering/smoothing problem.

There are four important factors in the covariance evolution, the: (i) initial condition $\mathbf{P}(0)$; (ii) effects of the deterministic model dynamics on the covariance, first two terms in the RHS of Eq. (4a); (iii) model uncertainties which increase variance, last term in Eq. (4a); and (iv) data impacts which reduce variance, Eq. (4b). A few oceanic uncertainty problems can be addressed with Eqs. (4a) and (4b). The first is the pure prediction problem, in real-time or in hindcast, which starts from realistic initial conditions $\mathbf{P}(0)[54,64]$. The second is the reanalysis or full assimilation problem, including possibly both filtering and smoothing, which computes uncertainties after the data collection and can lead to close to stationary errors if the observation system is well chosen/adapted. The third is the predictability problem which estimates the time by which the limit of predictability has been reached: i.e., the $\operatorname{tr}(\mathbf{P})$ has grown to be as large as that of the variability (entropy-based criteria are also used [42]). Initial errors are then set small and random, white or red in space.

\subsubsection{Stochastic forcing}

The stochastic forcings in the model and parameter equations (1) and (2) were chosen additive, uncorrelated to state variables, but correlated in time and space, because the statistics of several natural processes can be approximated this way $[37,27,26]$. Since parameters enter model equations (Eqs. (1a) and (1c)), this formalism also includes a type of multiplicative noise. To arrive to Eqs. (1a) and (2a), the deterministic ocean dynamics and parameter evolution are first modified and forced by noise processes correlated in time and space. For the spatially discrete, augmented state vector $\mathbf{x}$, this leads

$$
\mathrm{d} \mathbf{x}=\mathscr{M}(\mathbf{x}, t)+\mathbf{B}(t) \mathrm{d} \tilde{\mathbf{w}}
$$

The tilde on d $\tilde{\mathbf{w}}$ denotes the stochastic forcing correlated in time. The matrix $\mathbf{B}(t)$ in Eq. (5) leads to spatial covariances $\mathbf{B}(t) \mathbf{B}(t)^{T} \geqslant 0$. It aims to model the spatial correlations of sea processes neglected or not well represented in the imperfect deterministic dynamics. The time dependence allows non-stationary statistics (as for most ocean processes), but does not allow this statistics to be a function of the values of $\mathbf{x}$ itself.

Mathematically, the time-correlated noise processes $\mathrm{d} \tilde{\mathbf{w}}$ in Eq. (5) is modeled by differential equations excited by Gaussian noise white in time $\mathbf{w}$,

$$
\mathrm{d} \tilde{\mathbf{w}}=\mathbf{g}(\tilde{\mathbf{w}}, t)+\tilde{\mathbf{B}}(t) \mathrm{d} \mathbf{w}
$$

By a second state vector augmentation, e.g. [27], Eqs. (5) and (6) are combined into a single equation for the joint state vector,

$$
\mathrm{d} \mathbf{x}=\mathscr{M}(\mathbf{x}, t)+\mathbf{B}(t) \mathrm{d} \mathbf{w},
$$


280 where up to dwambols of Eq. (5) have been formally kept to ease notation. Defining $\mathrm{d} \boldsymbol{\eta} \doteq \mathbf{B}(t) \mathrm{d} \mathbf{w}$ in Eq. (7) 281 with $\mathbf{Q}(t)=\mathbf{B}(t) \mathbf{B}(t)^{\mathrm{T}}$ leads to Eq. (2a).

The efficient modeling of the time-space noise covariances in Eqs. (5) and (6), according to specific uncer-

284

285

286

287

288

289

290

291

292

293

294

295 tain sea-processes, is challenging. There are essentially three approaches. The "empirical" choice uses the misfits between deterministic model forecast and observations, and organizes and maps these misfits back to the state space [12], possibly using a dominant singular value decomposition. The "analytical" derives stochastic equations for the most energetic deficient processes of the dynamical model (e.g., PE). The "numerical" utilizes notions related to stochastic optimals [22] to estimate what we call model error optimals. Presently, our choice is based on a simple, zeroth order version of the analytical approach, with model coefficients empirically estimated from observations. Details are outlined in Appendix A.2.

Importantly, what is modeled as a deterministic process or a stochastic process depends on the time and space scales contained in the scale window of interest (Section 1), or in other words, on the applications and questions posed [28]. In ocean modeling, errors due to missing processes and approximate parameterizations, boundary conditions and initial conditions are usually much larger than uncertainties due the numerical solutions of the model equations. For the incorporation and propagation of numerical uncertainties in computational fluid dynamics using polynomial chaos ideas, we refer to [41].

\subsection{Computational method}

Error subspace statistical estimation (ESSE, [48,55]) aims to characterize and predict the largest of the uncertainties governed by Eqs. (3) and (4). It is based on evolving an error subspace, of variable size, that spans and tracks the scales and processes where the dominant errors occur. Model and data are combined in accord with these predicted dominant uncertainties. Mathematically, it is the DA criterion (Eq. (1d)) that sets the definition of the subspace. The suboptimal truncation of errors in the full space is then optimal. For minimum error variance estimation, the subspace is defined by the ordered eigen decomposition of a normalized form of the multivariate $\mathbf{P}$, denoted here by $\mathbf{P}_{k}^{p}$ for a rank $p$ (Eq. (8)). The corresponding DA criterion is Eq. (9).

$$
\begin{aligned}
& \text { Er. Subpace: }\left\{\mathbf{P}_{k}^{p}=\mathbf{E}_{k} \boldsymbol{\Pi}_{k} \mathbf{E}_{k}^{\mathrm{T}} \text { with } \operatorname{rank}\left(\mathbf{E}_{k}\right)=p \mid \min _{\boldsymbol{\Pi}_{k}, \mathbf{E}_{k}}\left\|\mathbf{P}_{k}-\mathbf{P}_{k}^{p}\right\|\right\} \\
& \text { Min. Er. Var.: }\left\{\widehat{\mathbf{x}}_{k} \mid \min _{\widehat{\mathbf{x}}_{k}} J_{k}=\operatorname{tr}\left[\mathbf{P}_{k}^{p}(+)\right] \text { using }\left[\mathbf{y}_{0}^{\mathrm{o}}, \ldots, \mathbf{y}_{k}^{\mathrm{o}} / \mathbf{y}_{N}^{\mathrm{o}}\right]\right\}
\end{aligned}
$$

Note that different norms in Eq. (8) lead to different subspaces. In ocean predictions, errors in the initial conditions and models are usually large and, as long as the normalization employed is meaningful, its details have a limited influence on the DA results. The components and time-workflow of the ESSE system are illustrated in Fig. 1. The sequential computations are currently based on $\mathbf{P}_{k}^{p}$ for the data update and on an adaptive, stochastic ensemble scheme for a nonlinear prediction of $\mathbf{P}_{k}^{p}$, aiming to approximate Eqs. (4a) and (4b).

The multivariate error subspace (ES) is initialized based on a decomposition on multiple scales of uncertainties [51,54], using historical data and dynamics. Uncertainties at $t_{0}$ are assumed to be the dominant uncertain variability in the initial mean state $\widehat{\mathbf{x}}_{0}$. For parts of this variability, some data are often available. These "observed portions" of $\mathbf{P}_{0}^{p}$ are directly specified from differences between $\widehat{\mathbf{x}}_{0}$ and data, or from a statistical model fit to these differences. The "non-observed" portions are then computed by dynamical inference, using an ensemble of model integrations (Eq. (2a)). Dynamical cross-covariances adjust these unknown portions to the specified observed ones. The result is an estimate of the initial decomposition $\mathbf{E}_{0}, \boldsymbol{\Pi}_{0}$ (Fig. 1: left oval). To create initial states, a random combination of these error modes is added to $\widehat{\mathbf{x}}_{0}$. If the result passes dynamical and data constraints, it is a possible state.

At time $t_{k}, \widehat{\mathbf{x}}_{k}(+)$ is perturbed (Eq. (11)) using $\mathbf{E}_{k}(+)$ with adequate random coefficients $\pi_{k}^{j}(+)$ based on $\boldsymbol{\Pi}_{k}(+)$ and dynamical constraints [48]. Random white noise $\mathbf{n}_{k}^{j}$ is also added to model the truncated tail of the error spectrum. To evolve fields and uncertainties up to $t_{k+1}$ (Fig. 1: central oval), a central forecast (Eq. (10a)) and an ensemble of $j=1, \ldots, q$ stochastic ocean model integrations are carried out (Eq. (12)), starting from the perturbed states $\mathbf{x}_{k}^{j}(+)$. The forcings $\mathscr{E}\left\{\mathrm{d} \boldsymbol{\eta}(t) \mathrm{d} \boldsymbol{\eta}^{\mathrm{T}}(t)\right\} \doteq \mathbf{Q}(t) \mathrm{d} t$ are defined in Appendix A.2. The 


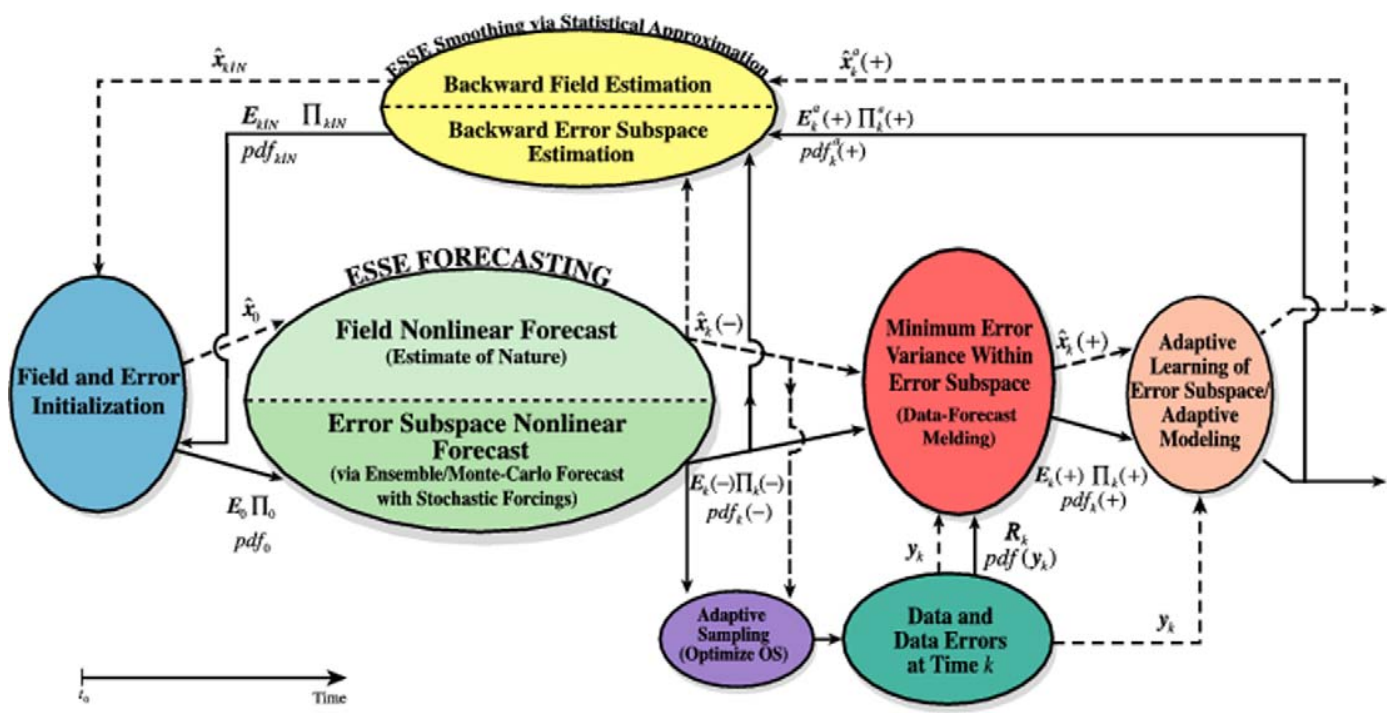

Fig. 1. Seven main components of the present error subspace statistical estimation system and its schematic workflow with time.

error subspace forecast (Eq. (13)) is computed from this ensemble. The matrix $\mathbf{M}_{k+1}(-)=\left[\widehat{\mathbf{x}}_{k+1}^{j}(-)-\right.$ $\widehat{\mathbf{x}}_{k+1}(-)$, difference between $q$ realizations and an estimate of the conditional mean, e.g. $\widehat{\mathbf{x}}_{k+1}^{\text {em }}(-)$ (Eq. $(10 \mathrm{~b})$ ), is computed. It is then normalized and decomposed (Eq. (13)) into $\boldsymbol{\Pi}_{k+1}(-) \doteq \frac{1}{q} \boldsymbol{\Sigma}_{k+1}^{2}(-)$ and $\mathbf{E}_{k+1}(-)$ of rank $p \leqslant q$ by singular value decomposition (the operator $\operatorname{SVD}_{p}(\cdot)$ selects the rank- $p \mathrm{SVD}$ ). The ensemble size is increased and ultimately controlled by convergence criteria. The coefficient $\rho$ used here (Eq. (14)) in the applications (Section 4) measures the similarity between two subspaces of different sizes. A "previous" estimate $(\mathbf{E}, \boldsymbol{\Pi})$ of rank $p$ and "new" estimate $(\widetilde{\mathbf{E}}, \widetilde{\boldsymbol{\Pi}})$ of rank $\tilde{p} \geqslant p$ are compared, using singular values to weight singular vectors. In Eq. (14), $\alpha$ is a scalar $(1-\epsilon \leqslant \alpha \leqslant 1)$ chosen by the user, $\sigma_{i}(\cdot)$ selects the singular value number $i$ and $k=\min (\tilde{p}, p)$. When $\rho$ is close enough to one, the two subspaces are similar in the variance explained sense. The resulting $(\widetilde{\mathbf{E}}, \widetilde{\mathbf{\Pi}})$ is then chosen as ES forecast for $t_{k+1}: \boldsymbol{\Pi}_{k+1}(-), \mathbf{E}_{k+1}(-)$. The dimensions of the ensemble $(q)$ and ES $(p)$ hence vary with time, in accord with data and dynamics.

Central fcst: $\widehat{\mathbf{x}}_{k+1}^{\mathrm{cf}}(-) \mid \mathrm{d} \widehat{\mathbf{x}}=\mathscr{M}(\widehat{\mathbf{x}}, t) \mathrm{d} t \quad$ with $\widehat{\mathbf{x}}_{k}=\widehat{\mathbf{x}}_{k}(+)$

Ens. mean: $\widehat{\mathbf{x}}_{k+1}^{\mathrm{em}}(-) \doteq \mathscr{E} q\left\{\widehat{\mathbf{x}}_{k+1}^{j}(-)\right\}$

ES In. Cond.: $\widehat{\mathbf{x}}_{k}^{j}(+)=\widehat{\mathbf{x}}_{k}(+)+\mathbf{E}_{k}(+) \boldsymbol{\pi}_{k}^{j}(+)+\mathbf{n}_{k}^{j}, \quad j=1, \ldots, q$

Ens. Fcst: $\widehat{\mathbf{x}}_{k+1}^{j}(-) \mid \mathrm{d} \widehat{\mathbf{x}}^{j}=\mathscr{M}\left(\widehat{\mathbf{x}}^{j}, t\right) \mathrm{d} t+\mathrm{d} \boldsymbol{\eta} \quad$ with $\widehat{\mathbf{x}}_{k}^{j}=\widehat{\mathbf{x}}_{k}^{j}(+)$

ES Fcst: $\mathbf{M}_{k+1}(-)=\left[\widehat{\mathbf{x}}_{k+1}^{j}(-)-\widehat{\mathbf{x}}_{k+1}(-)\right]$

$$
\left\{\boldsymbol{\Sigma}_{k+1}(-), \mathbf{E}_{k+1}(-) \mid \mathbf{S V D}_{p}\left(\mathbf{M}_{k+1}(-)\right)=\mathbf{E}_{k+1}(-) \boldsymbol{\Sigma}_{k+1}(-) \mathbf{V}_{k+1}^{\mathrm{T}}(-)\right\}
$$

Conv. Crit.: $\rho=\frac{\sum_{i=1}^{k} \sigma_{i}\left(\boldsymbol{\Pi}^{\frac{1}{2}} \mathbf{E}^{\mathrm{T}} \widetilde{\mathbf{E}} \widetilde{\boldsymbol{\Pi}}^{\frac{1}{2}}\right)}{\sum_{i=1}^{\tilde{p}} \sigma_{i}(\widetilde{\boldsymbol{\Pi}})} \geqslant \alpha$

Once the ES forecast is completed, the data and their error estimates (Fig. 1: second bottom oval) are employed. Data can be acquired by adaptive sampling (Fig. 1: first bottom oval), e.g. predict the observations that will reduce errors the most [52]. Once data are available, data-forecast misfits are used to correct the predicted state by multivariate minimum error variance estimation in the ES (Fig. 1: middle oval). The results are Eqs. (15)-(18), where $\mathbf{H}^{p} \doteq \mathbf{H E}$ - and the subscript $k+1$ has been omitted. Importantly, Eqs. (15)-(18) are only a linear approximation of Eq. (4b). Outputs are the filtering estimates: the a posteriori fields $\mathbf{x}(+)$ and a posteriori error subspace covariance, i.e., $\mathbf{E}_{+}, \boldsymbol{\Pi}(+)$. Filtering covariance estimates can also be obtained from a direct update of the SVD of the ensemble spread (see [48]). 
State Upd.: $\widehat{\mathbf{x}}(+)=\widehat{\mathbf{x}}(-)+\mathbf{K}^{p}\left(\mathbf{y}^{\mathrm{o}}-\mathscr{H}(\widehat{\mathbf{x}}(-))\right)$

ES Optimal Gain: $\mathbf{K}^{p}=\mathbf{E}_{-} \boldsymbol{\Pi}(-) \mathbf{H}^{p^{\mathrm{T}}}\left(\mathbf{H}^{p} \boldsymbol{\Pi}(-) \mathbf{H}^{p^{\mathrm{T}}}+\mathbf{R}\right)^{-1}$

$$
\text { ES Cov. Upd.: } \mathbf{L} \Pi(+) \mathbf{L}^{\mathrm{T}}=\boldsymbol{\Pi}(-)-\boldsymbol{\Pi}(-) \mathbf{H}^{p^{\mathrm{T}}}\left(\mathbf{H}^{p} \boldsymbol{\Pi}(-) \mathbf{H}^{p^{\mathrm{T}}}+\mathbf{R}\right)^{-1} \mathbf{H}^{p} \boldsymbol{\Pi}(-)
$$

356

357

358

359

360

361

362

363

$$
\mathbf{E}_{+}=\mathbf{E}_{-} \mathbf{L}
$$

Ultimately, the smoothing via ESSE [48] is carried out backward in time (Fig. 1: top oval) to correct, based on future data, the past coupled fields and uncertainties (Eqs. (23)-(26)). Starting from the filtering estimate, a statistical approximation to the forward integration of the dynamical model between two data times $t_{k-1}$ and $t_{k}$ is derived. The approximation is a backward statistical linearization (Eq. (24)) based on the a posteriori ES at $t_{k-1}$ and nonlinear ES forecast at $t_{k}$. This backward rule is then used to minimize the smoothing DA criterion, leading to the smoothing estimate (Eq. (23)) and its errors (Eqs. (25) and (26)). Carrying out the smoothing process recursively up to $t_{0}$ leads to the smoothed initial fields $\mathbf{x}_{0 / N}$ and errors, e.g., $\mathbf{E}_{0 /}$ ${ }_{N}, \Pi_{0 / N}$.

Filtering Est.: ESSE filtering during $\left[t_{0}, t_{N}\right]$

Sm. Est.: $\widehat{\mathbf{x}}_{k / N}=\widehat{\mathbf{x}}_{k}(+)+\mathbf{L}_{k}^{p}\left(\widehat{\mathbf{x}}_{k+1 / N}-\widehat{\mathbf{x}}_{k+1}(-)\right)$ with $\widehat{\mathbf{x}}_{N / N}=\widehat{\mathbf{x}}_{N}(+)$

Sm. Gain: $\mathbf{L}_{k}^{p}=\mathbf{E}_{k}(+) \boldsymbol{\Gamma}_{k} \mathbf{E}_{k+1}^{\mathrm{T}}(-) \quad$ and $\quad \boldsymbol{\Gamma}_{k}=\boldsymbol{\Sigma}_{k}(+) \mathbf{V}_{k}^{\mathrm{T}}(+) \mathbf{V}_{k+1}(-) \boldsymbol{\Sigma}_{k+1}^{-1}(-)$

Sm. Err. Cov.: $\mathbf{H}_{k} \boldsymbol{\Pi}_{k / N} \mathbf{H}_{k}^{\mathrm{T}}=\boldsymbol{\Pi}_{k}(+)+\boldsymbol{\Gamma}_{k}\left(\boldsymbol{\theta}_{k+1} \boldsymbol{\Pi}_{k+1 / N} \boldsymbol{\theta}_{k+1}^{\mathrm{T}}-\boldsymbol{\Pi}_{k+1}(-)\right) \boldsymbol{\Gamma}_{k}^{\mathrm{T}}$

$$
\mathbf{E}_{k / N}=\mathbf{E}_{k}(+) \mathbf{H}_{k}, \quad \text { with } \quad \boldsymbol{\theta}_{k+1} \doteq \mathbf{E}_{k+1}^{\mathrm{T}}(-) \mathbf{E}_{k+1 / N}
$$

\subsection{Numerical schemes and implementation}

The ESSE numerical schemes corresponding to Fig 1 are relatively complex. Challenges arise due to the: varied ocean geometries; measurement models and diverse data types and locations; and, dynamical models, with multiple state variables and their stochastic forcing. However, many computations are based on linear algebra which allows the use of efficient community packages. Focusing on the implementation, the main version of the system is based on Fortran codes which utilize packages such as Lapack for optimum performance (on distributed and parallel computers). These codes are managed based on workflows. For example, let us consider the workflow of the uncertainty prediction module. Such predictions currently involve an initialization software to estimate $\mathbf{P}_{0}^{p}$. A script is then executed which manages several codes for: (i) the perturbation of the initial mean state, (ii) the subsequent ensemble of stochastic PE model runs, including the successive computations of the SVD of the ensemble spread until a convergence criterion is satisfied, and (iii) the data assimilation. Of course, such script workflows contain different types of variables, including limit values, constants and evolving parameters, which need to be selected by the user. In the future, web-based systems will facilitate the use of such workflows [20]. Considering computational cost, ESSE 
391 is on average about $10^{3}-10^{4}$ times less expensive than classic linear schemes (e.g., Kalman filter/smoother). 392 Without such reductions, real-time and realistic predictions of uncertainties are not feasible with today's 393 computers.

394 Of course, there are several options and heuristics to the ESSE scheme. They include: the parameters setting 395 the amplitude of the initial error conditions; the definition of the ocean forecast from the ensemble; the adap396 tation of the statistics of $\mathbf{n}_{k}^{j}$ based on observations; and, when too small ensemble sizes are used, the optional 397 reduction of error covariances at long distances, based on a Schur product with another covariance.

398

\section{Interdisciplinary applications}

The applications presented illustrate capabilities of the ESSE computational scheme for oceanic uncertainty estimation and prediction. Data properties and the specifics and scientific results of each application are not discussed in detail. Complete versions of ESSE were utilized in each application but only parts of the whole system are illustrated in each case, with limited methodological overlap. The first application focuses on the estimation of uncertainties for coupled physical-biogeochemical fields. The second outlines the transfer of oceanic physical uncertainties to acoustical fields and shows some physical-acoustical interactions. The last discusses real-time physical stochastic ensemble predictions, with assimilation of a wide range of measurement types.

\subsection{Uncertainty estimations for physical-biogeochemical fields in Massachusetts Bay}

Regardless of the computational method used for uncertainty quantification, a challenge for multivariate multi-dimensional fields is the visualization of uncertainties [15]. In addition to $p\left(\mathbf{x}, t \mid \mathbf{y}_{t_{-}}\right)$, the conditional PDF of the full discrete state $\mathbf{x}$ (Section 3.1), a PDF can be estimated for each element of $\mathbf{x}$. With the ESSE ensemble, an histogram can be formed at every position and time, for every variable. Fig. 2 illustrates the field of local PDFs of the surface velocity in Massachusetts Bay (Mass Bay) as hindcasted for September 2, 1998, based on 600 realizations. Mean velocity amplitudes and vectors are on Fig. 2a. Local PDF estimates at two critical point locations (point with mean velocity close to 0 ) are plotted in Fig. 2b. One of the results is that state variables are close to be Gaussian at some locations but not everywhere. Of course, there are multiple ways to visualize uncertainties in oceanic predictions [15]. Ensemble forecasts also need to be evaluated and several evaluation methods are now utilized (see [38,39]). They include rank histograms [2,32] or scalar Talagrand diagrams [75], multi-dimensional scaling schemes [74], minimum spanning tree histograms $[79,73]$ and bounding boxes [77]. Connections among visualization and evaluation schemes should in fact be useful for future research.

The above physical hindcast was initialized for August 20, 1998. Biogeochemical fields and uncertainties were also initialized for that period (see Section 3.2). To estimate the initial physical-biogeochemical covariance $\mathbf{P}_{0}^{p}$, models (Eq. (2a)) are needed because historical data sets with synoptic physical and biogeochemical measurements are limited. For Mass. Bay, the data available were profiles of temperature, salinity and chlorophyll- $a$, as well as a few coarse resolution profiles of nitrate and ammonium. The first two empirical orthogonal functions (EOFs) of these profiles are shown in Fig. 3. The first EOF is related to variations of $T$ and $S$ that are in opposition of phase (hence limited density variations) and to primary production dynamics $\left(\mathrm{NO}_{3}\right.$ and $(h l)$. The second EOF is linked to fuller biogeochemical dynamics and salinity variations. Vertical EOFs were combined with dominant horizontal analytical eigenfunctions (not shown) to lead to three-dimensional eigenvectors for $T, S, C h l, \mathrm{NO}_{3}$ and $\mathrm{NH}_{4}$, and their eigenvalues. This "observed" decomposition was then used to perturb $\widehat{\mathbf{x}}_{0}$ and estimate the "non-observed" variability by integration of the prognostic equations of the "non-observed" state variables (Appendix A). $\mathbf{P}_{0}^{p}$ is ultimately estimated from the resulting variability samples.

ESSE is started from this dynamically-adjusted $\mathbf{P}_{0}^{p}$. The convergence criterion $\rho \geqslant \alpha$ (Eq. (14)) with $\alpha=0.97$ was evaluated for every batch of 100 runs and was reached after 600 runs. The resulting uncertainty prediction is shown first (Fig. 4) by vertical sections across Mass Bay (Cape Cod to Cape Ann) in the forecast error standard deviation fields for $\mathrm{NO}_{3}, C h l, \mathrm{NH}_{4}$ and $D$. One can recognize effects of the dynamics (first two terms in the RHS of Eq. (4a)). For Chl, the uncertainty is largest above Stellwagen Bank, near its sub-surface 

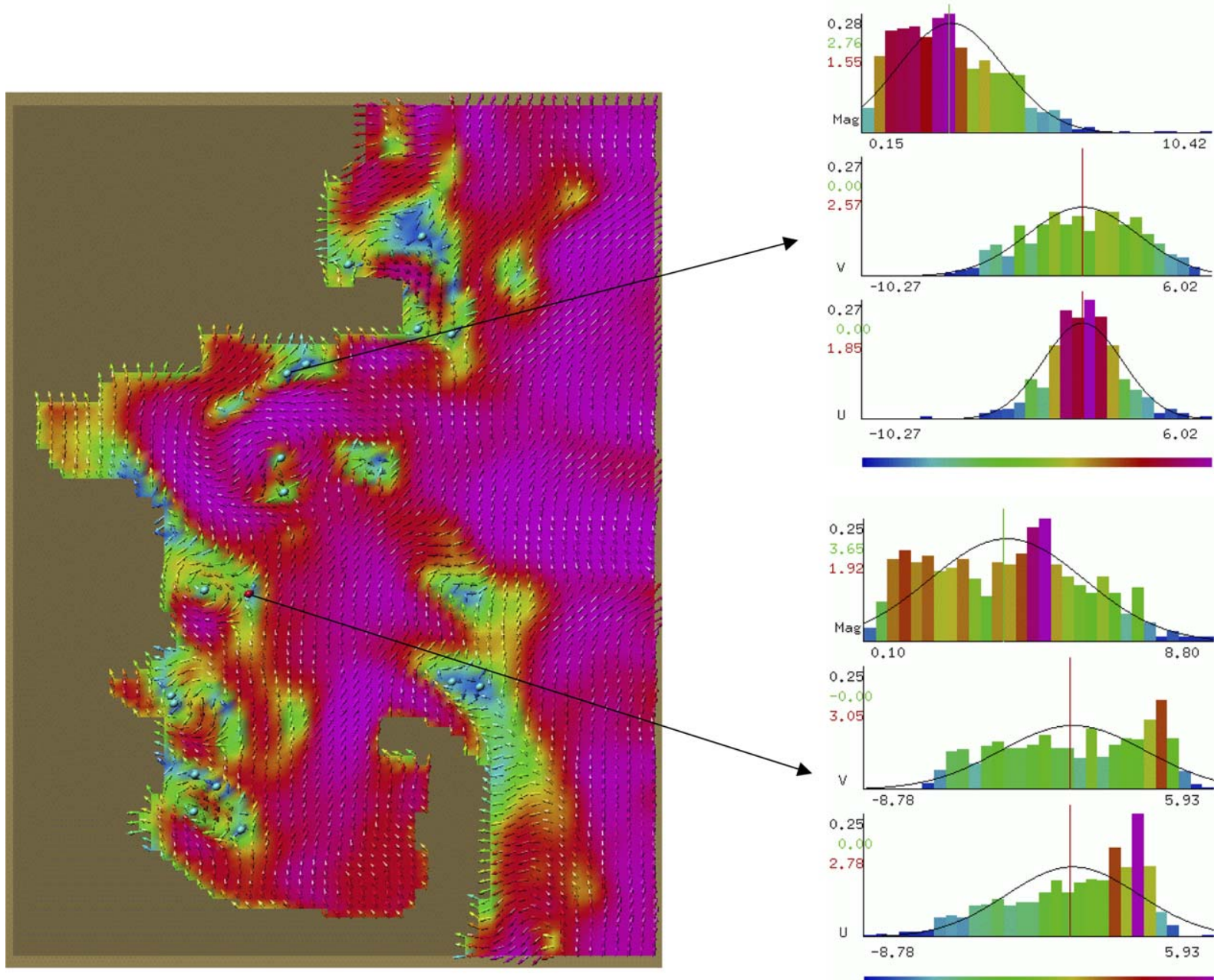

Fig. 2. Local PDF's of surface velocity, as estimated by ESSE for Massachusetts Bay on September 2, 1998, and illustrated by A. Love, W. Shen and A. Pang (UCSC). (a, left) Mean horizontal velocity magnitude $\left|\mathbf{u}_{h}\right|$ (color mapping, blue to red/purple) and directions (arrows). (b, right) Horizontal velocity PDFs (histograms) estimated based on the 600 ESSE ensemble members, at two locations. The PDF of $\left|\mathbf{u}_{h}\right|$ (top) and these of its two components $v$ and $u$ (below) are shown. Color on the histograms represents the PDF value ( $y$-axis), from blue to red/purple. The $x$-axis is in $\mathrm{cm} / \mathrm{s}$ (black numbers). For each histogram, a parametric fit to a target Gaussian PDF is shown (black curve); the red vertical line is the zero value line. The y-axis is the PDF value (black tick-marks). The green number is the mean $\left|\mathbf{u}_{h}\right|$ and the red number its standand deviation. (For interpretation of the references to colour in this figure legend, the reader is referred to the web version of this article.)

maxima. The $D$ uncertainties are also large on the northern side near Cape Ann due to $D$ sinking and to downwelling. For $\mathrm{NO}_{3}$, it is largest just below the largest $C h l$ uncertainties and along the coast at depth due to upwelling. Similar comments can be made for $\mathrm{NH}_{4}$.

The same vertical cross-section is now taken in error covariance field estimates (Fig. 5). Clockwise, the auto-covariance for $C h l$ is shown first, then cross-covariances between $C h l-\mathrm{NO}_{3}, C h l-S$ and $C h l-\hat{v}$ (see Appendix A.1 for definitions). The location of the maximum in the $C h l$ auto-covariance is the $C h l$ point with which all shown covariances are computed. Should a $C h l$ observation be made at this point, the four fields shown indicate how its ESSE assimilation would impact the $\mathrm{Chl}, \mathrm{NO}_{3}, \mathrm{~S}$ and $\hat{v}$ estimates. For each field, the location of the extrema are in accord with expected dynamics. Correlations are stronger with $\mathrm{Chl}$ and $\mathrm{NO}_{3}$ than with $\mathrm{S}$ and $\hat{v}$. A single $C h l$ observations at that location would have a smaller relative impact on these two physical fields. 

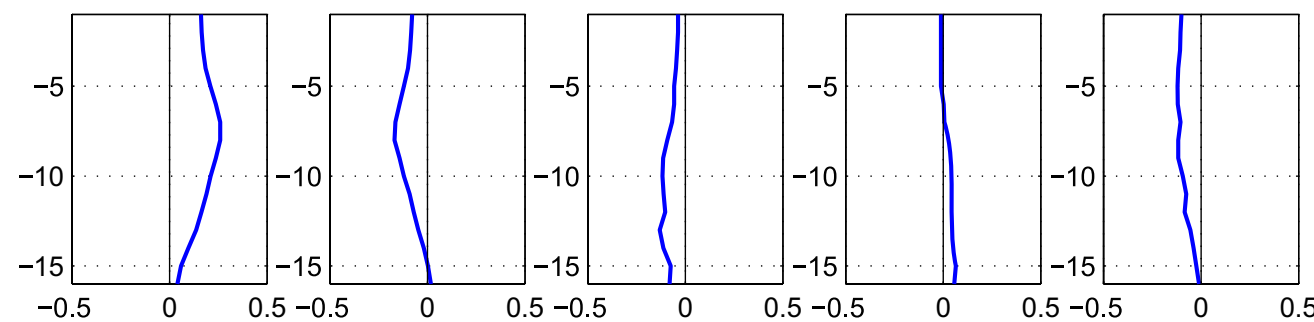

T comp. eof $1 \quad \mathrm{~S}$ comp. eof 1

NO3 comp. eof 1

$\mathrm{NH} 4$ comp. eof 1

CHL comp. eof 1

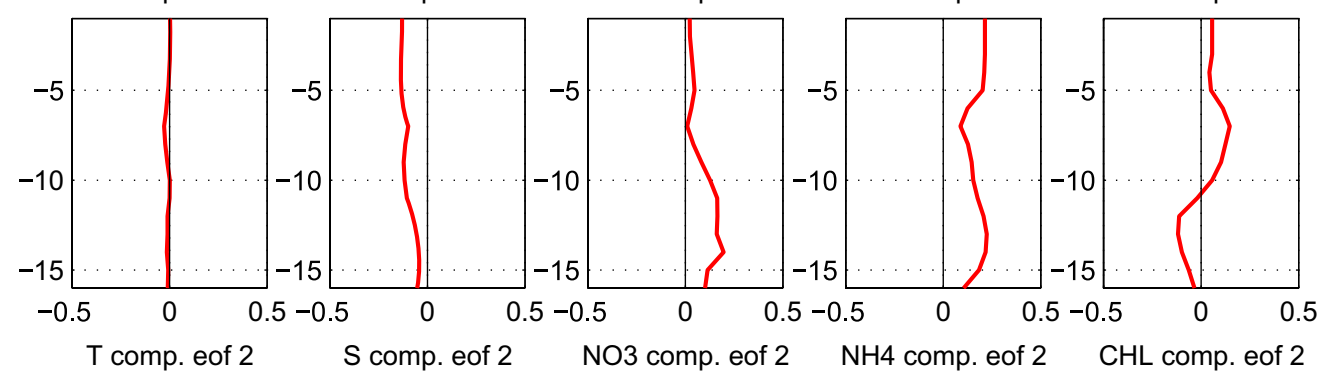

Fig. 3. First step in the initialization of physical-biogeochemical uncertainties for Mass Bay on August 20, 1998. Shown are the two dominant EOFs of non-dimensionalized synoptic historical profiles of temperature, salinity, nitrate, ammonium and chlorophyll- $a$, as a function of depth (model level, $y$-axis) and amplitude ( $x$-axis).
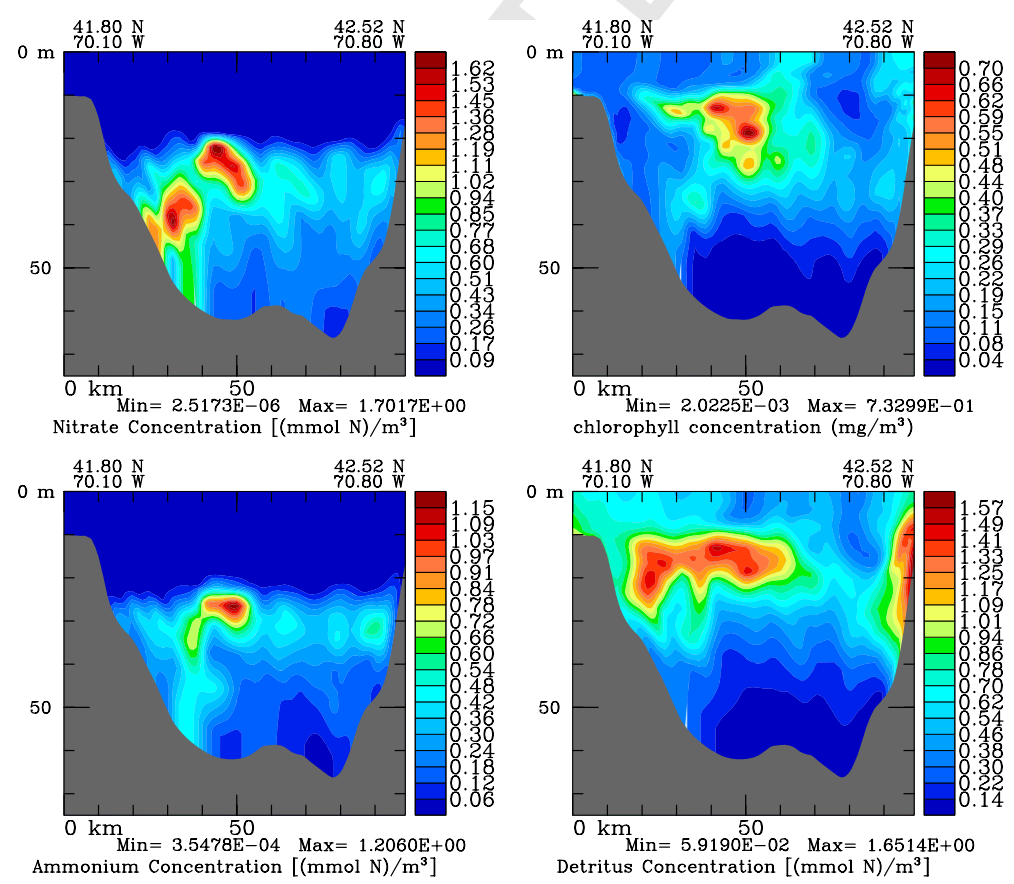

Fig. 4. Cross-sections in biogeochemical error standard deviation fields, hindcast for September 2, 1998 and computed using 600 ESSE ensemble members.

\subsection{Physical-acoustical uncertainties in the middle atlantic bight (MAB)}

The main oceanographic feature in the MAB shelfbreak is a mesoscale front of temperature, salinity and hence sound-speed, separating the shelf and slope water masses (Fig. 6a). The frontal system is variable on multiple scales. Atmospheric forcing, Gulf Stream rings, river inflows and buoyancy flow troughs, as well 

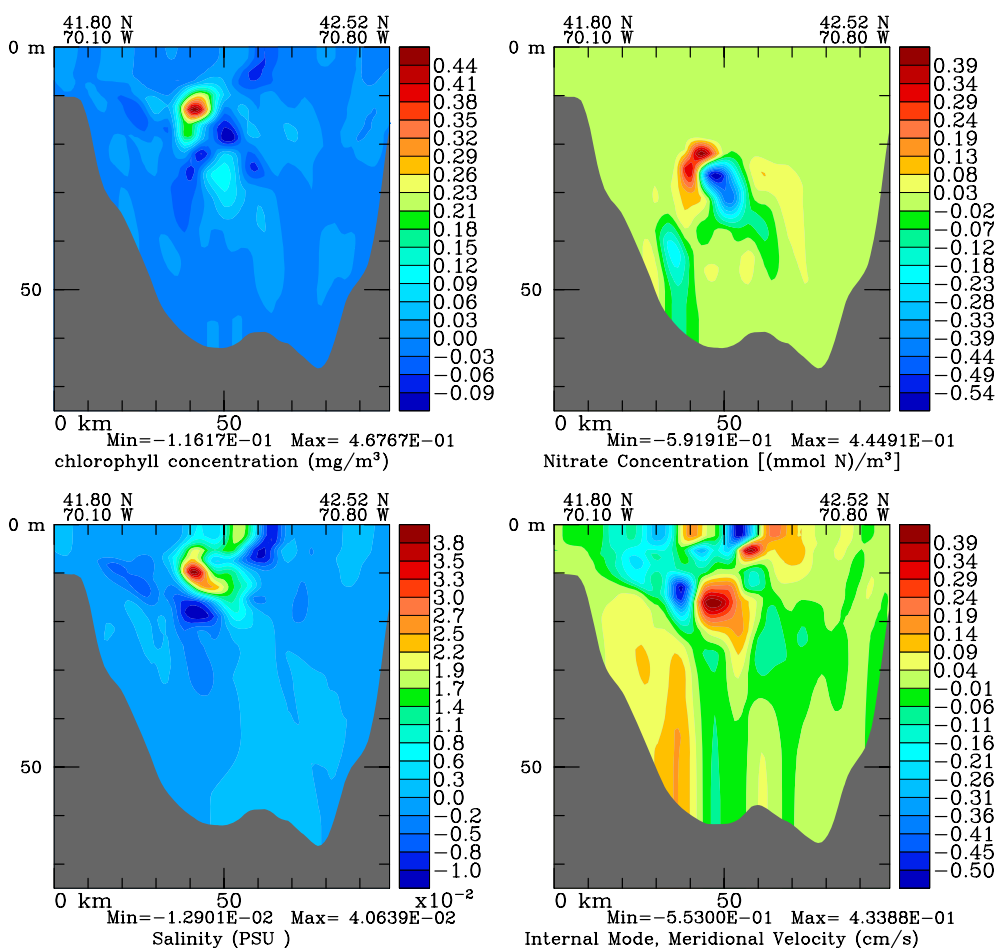

Fig. 5. Cross-sections in ESSE biogeochemical-physical error covariance fields, hindcast for September 2, 1998 and computed using 600 ESSE ensemble members. Clockwise, panels show the Chl-Chl covariance (the location of the maximum is the point with which all shown covariances are computed), $C h l-\mathrm{NO}_{3}$ covariance, $C h l-S$ covariance and $C h l-\hat{v}$ covariance. Each panel only gives the name and units of the field with which the $C h l$ covariance is computed.
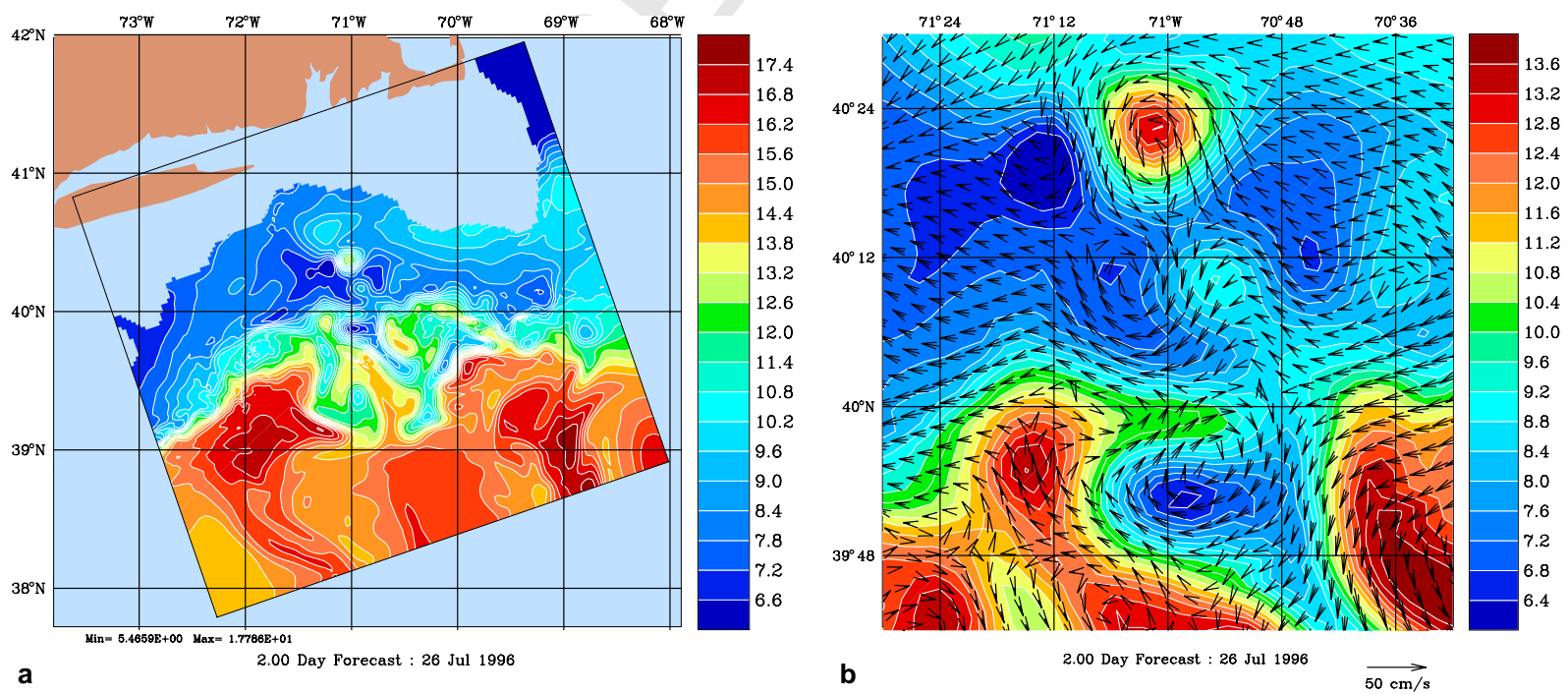

Fig. 6. HOPS simulation of PRIMER dynamics, with assimilation of all available physical data. (a) $50 \mathrm{~m}$ Temp. on July 26 in $387 \mathrm{~km}$ by $360 \mathrm{~km}$ domain (note large meander); (b) zoom in PRIMER acoustic domain ( $89 \mathrm{~km}$ by $85 \mathrm{~km}$ ), for $50 \mathrm{~m}$ Temp on Jul 26 , overlaid with velocity vectors.

454 as tides and internal waves, affect its dynamics. The main in situ data utilized were collected during July 26 to 455 August 4, 1996, over an intensive acoustic domain (Fig. 6b), as part of the ONR Shelfbreak PRIMER Exper456 iment [59]. Substantial dynamical model tuning was carried-out to achieve reasonable physical-acoustical sim- 

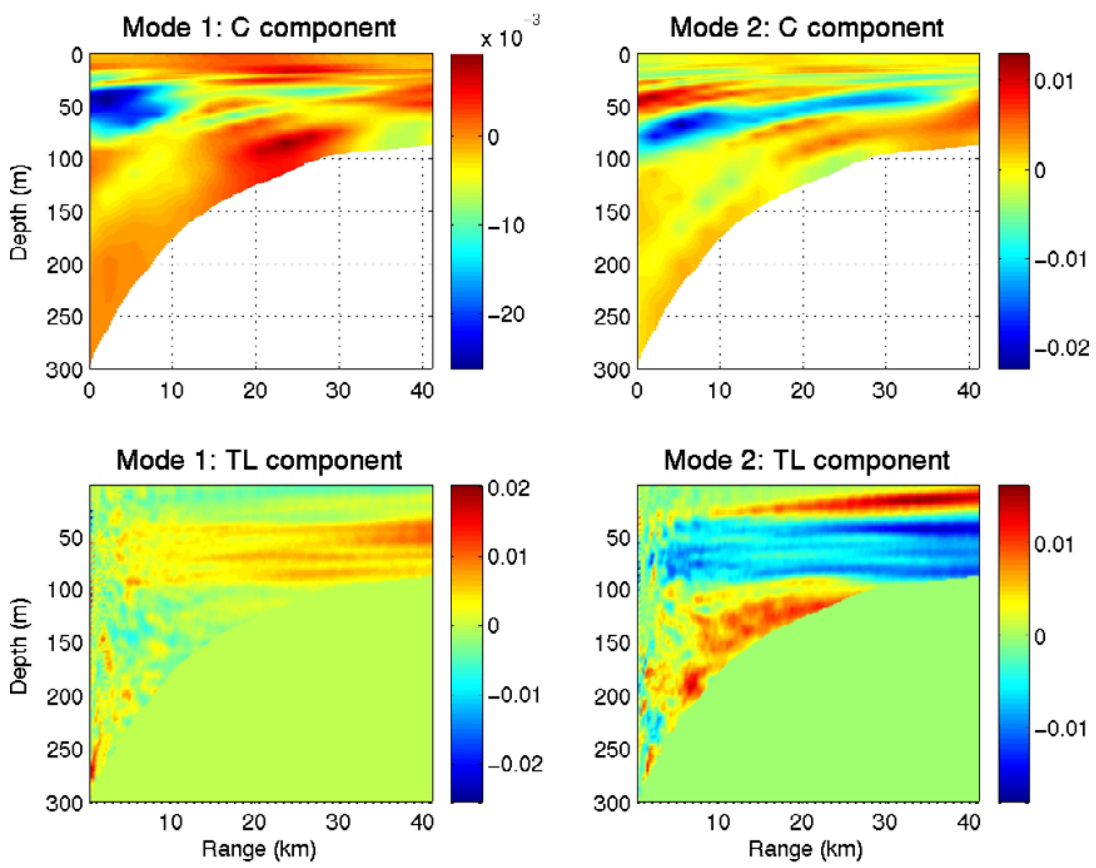

Fig. 7. Eigenmodes of the normalized physical-acoustical error covariance on July 26, along the main acoustic vertical section. The first mode is on the right, the second on the left. The upper fields show the sound-speed component, the lower fields the broadband TL component.

ulations. ESSE is started for July 8, based on synoptic National Marine Fisheries surveys and other historical data. The central forecast (Eq. (10a)) on July 26 is illustrated in Fig. 6, by horizontal maps of the $50 \mathrm{~m} T$ overlaid with $\mathbf{u}_{h}$. Note the large meander in Fig. 6a and (sub)-mesoscale variability in Fig. 6b which is important in summer, as anticipated from idealized simulations [49]. Interestingly, there are similarities between this submesoscale eddy field and open-ocean mesoscale eddies.

Sound-propagation studies often focus on vertical sections. ESSE ocean physics uncertainties on July 26 are transferred to acoustical uncertainties along such a section across the shelfbreak (Fig. 7). Time is fixed and an acoustic broadband transmission loss (TL) field is computed for each ocean realization. The $450 \mathrm{~Hz}$ sound source is at $300 \mathrm{~m}$ depth, near the deepest point on the slope. The coupled physical-acoustical covariance $\mathbf{P}^{p}$ for the section is computed and non-dimensionalized. Its first two dominant eigenvectors are shown in Fig. 7. The first uncertainty mode (left panels) mostly corresponds to possible shifts in the frontal shape, with their acoustic TL responses above the source and in the cold channel on the shelf. The second mode contains an opposition to the first and a tilt of the surface thermocline, leading to less/more loss in the cold channel and more/less loss in the surface and bottom ducts. Importantly, these modes (first two columns in $\mathbf{E}(-)$ ) are used for coupled physical-acoustical assimilation of hydrographic and TL data (not shown).

\subsection{Real-time stochastic ensemble predictions and DA in the Monterey Bay region}

A large ONR-sponsored, multi-institution coastal exercise, the Autonomous Ocean Sampling Network-II (AOSN-II, [1]), occurred in August 2003 in the Monterey Bay region off central California. The main features in the region are schematized in Fig. 8. ESSE was utilized to carry out nonlinear ensemble forecasts of physical fields and uncertainties of 2-3 days duration, assimilate various data types (ships, AUVs, gliders, aircraft, and satellites) and provide suggestions for adaptive sampling and guide dynamical investigations. 


\section{REGIONAL FEATURES of Monterey Bay and California Current System and Real-time Modeling Domains (AOSN2, 4 Aug. - 3 Sep., 2003)}
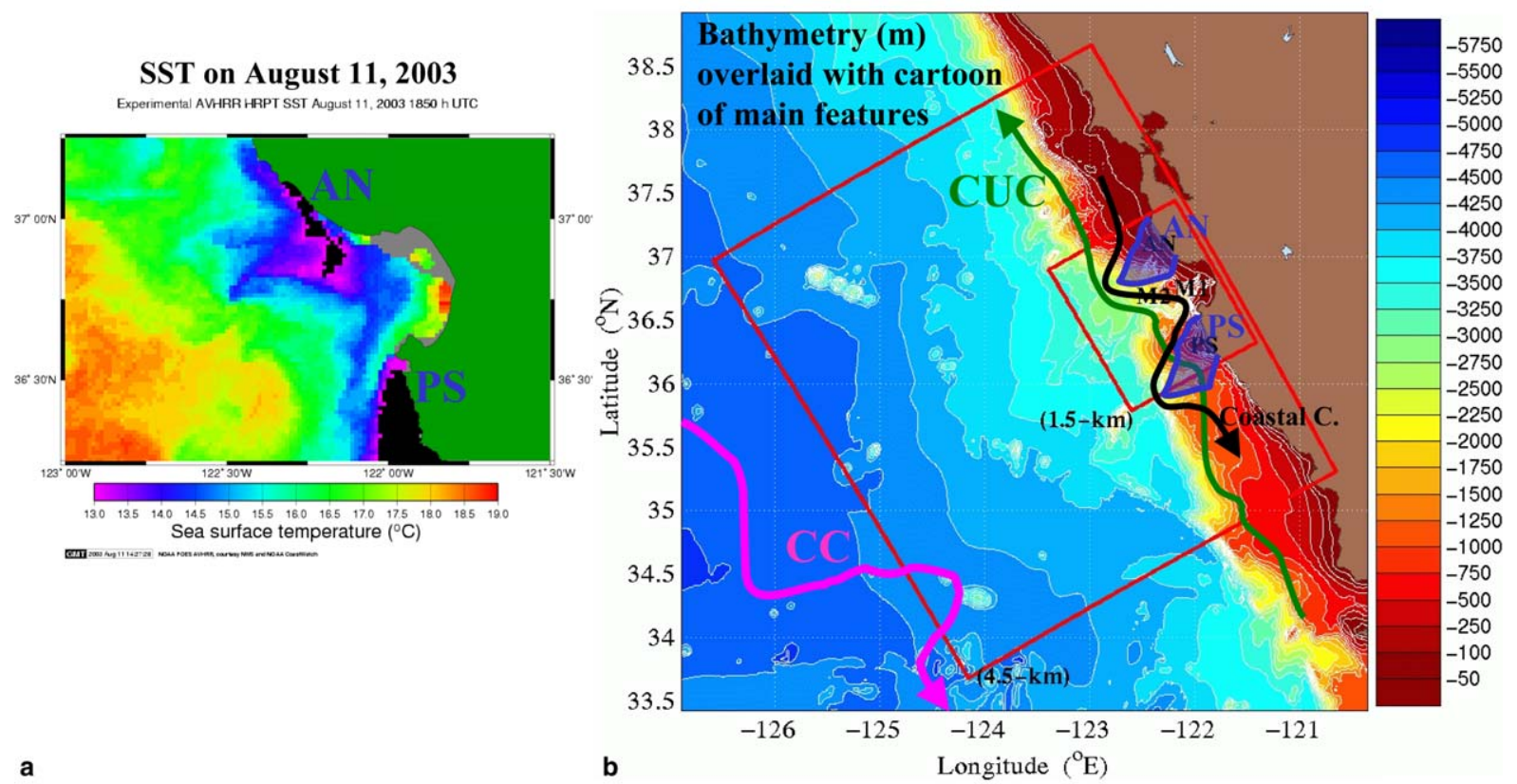

a

\section{REGIONAL FEATURES}

- Upwelling centers at Pt AN/ Pt Sur:

.Upwelled water advected equatorward and seaward

- Coastal current, eddies, squirts, filam., etc:....Upwelling-induced jets and high (sub)-mesoscale var. in CTZ

- California Undercurrent (CUC):. Poleward flow/jet, $10-100 \mathrm{~km}$ offshore, $50-300 \mathrm{~m}$ depth

- California Current (CC): Broad southward flow, 100-1350km offshore, $0-500 \mathrm{~m}$ depth

Fig. 8. Regional features of Monterey Bay and California Current System (CCS). (a) SST during upwelling event. (b) Modeling domains and cartoon of principal regional features with their main properties, as synthesized from the AOSN-II data-driven simulations (4 August to 3 September, 2003) and literature.

Error standard deviation values for the surface temperature are shown in Fig. 9. The onset of upwelling, sustained upwelling and relaxation conditions were captured, together with mesoscale variabilities and impacts of dynamics on uncertainties. In particular, field and error evolutions (Fig. 9) indicated that, during relaxation events, energy transfer occurred in the upper-layers, from atmospheric-driven large-scales to mesoscales. The build-up of larger-scale uncertainties during the upwelling periods is also visible in Fig. 9. Evaluations of real-time forecasts indicated relatively good agreement between data-model misfits and forecast uncertainties.

The effects of the stochastic forcings (Appendix A.2) are illustrated in Fig. 10. A deterministic and stochastic simulation are started from the same initial conditions and their differences after 1-day of integration are illustrated. The difference maps at $30 \mathrm{~m}$ (top of Fig. 10) clearly show that sub-grid-scales and sub-mesoscale variability can be statistically depicted by the stochastic terms. Transfer of un-resolved energies to resolved deterministic dynamical uncertainties also occurs. The differences in the vertical (bottom of Fig. 10) show effects of the vertically-varying amplitudes of the stochastic forcings, computed based on data misfits and geostrophic balance. $T$ and $S$ uncertainties are concentrated around the thermocline and halocline while $u$ and $v$ uncertainties are more slowly decaying in the upper $200 \mathrm{~m}$ depth.

496 ner. If the computation of the secondary variables is considered exact, it simply involves their evaluations for 497 each ESSE ensemble member. For example, the uncertainty on Lagrangian Coherent Structures (LCS) was 498 computed with Lekien [57] and is illustrated in Fig. 11, for the August 26-29 upwelling period in 2003. 


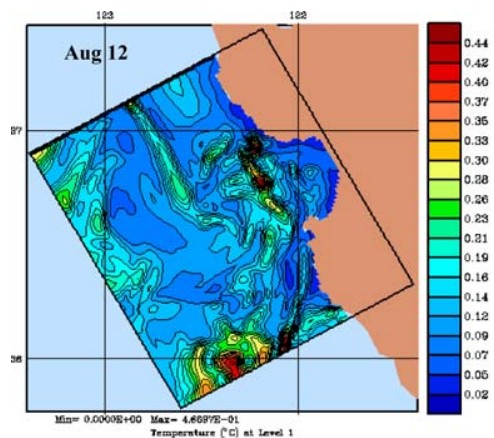

Start of Upwelling

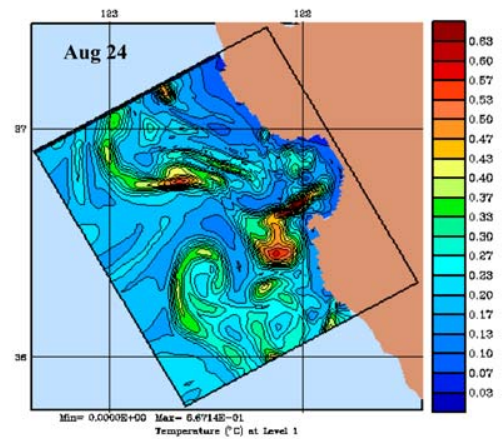

End of Relaxation
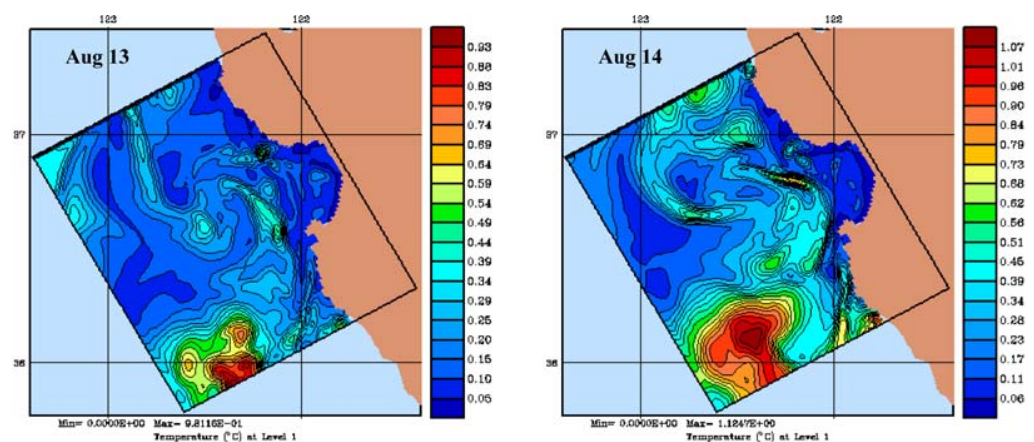

First Upwelling period
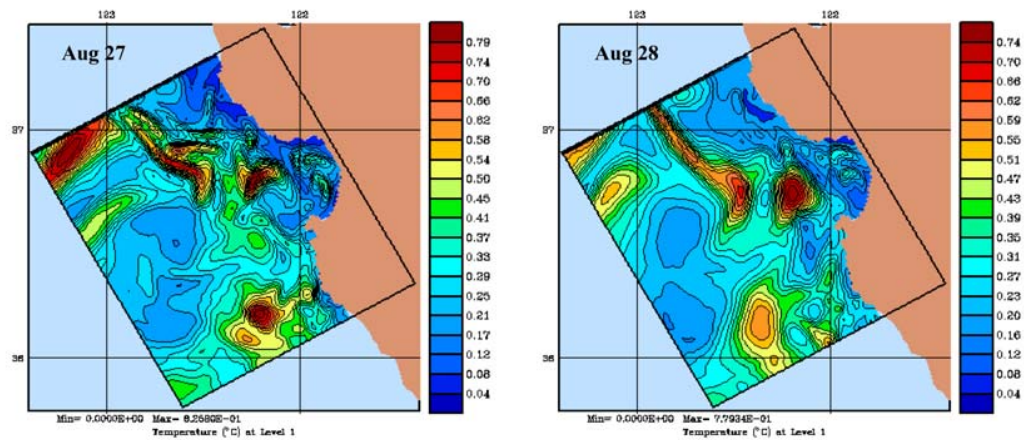

Second Upwelling period

Fig. 9. Sample of real-time ESSE forecasts of surface temperature error standard deviations $\left({ }^{\circ}\right)$.

499 The mean direct L yapounov exponent (DLE, [31]) and the corresponding LCS estimates are shown in 500 Fig. 11a, the error standard deviation of the DLE in Fig. 11b.

\section{5. Relationships to other approaches and promising directions}

There are several DA techniques for oceanic and atmospheric applications, see [70,40, and references therein]. However, few DA systems provide comprehensive and realistic predictions of uncertainties. ESSE is one of them and it has been applied with real ocean data first in the Strait of Sicily [50,53]. Related ensemble data assimilation studies, e.g. [21,63], have also started to be used in real-time in other ocean regions. In atmospheric applications, ensemble forecasting has been utilized for uncertainty predictions for some time [19] and realistic ensemble DA for weather prediction is now being investigated.

In some sense, ESSE combines DA with: (i) PODs or Karhunen-Loeve (KL) expansions [72,35]; (ii) timevarying basis functions, (iii) multi-scale initializations [51]; and (iv), stochastic ensemble predictions. It extends classic orthogonal basis/subspace decompositions that are fixed in time (pre-assigned functions $\phi_{k}(\mathbf{x})$, e.g. EOFs) to a dynamic data-driven low-dimensional representation of the error space. An ESSE decomposition can be written as

$$
\mathbf{x}(\mathbf{r}, t ; \theta)=\widehat{\mathbf{x}}(\mathbf{r}, t)+\sum_{i=1}^{p} \sqrt{\lambda_{i}(t)} \phi^{e}(\mathbf{r}, t) \zeta_{i}(\theta)
$$

where $\mathbf{x}$ is the ocean state, a function of space $\mathbf{r}=(x, y, z)$, time and random coefficients $\theta$; $\widehat{\mathbf{x}}$ is the mean estimate; $\lambda_{i}(t)$ the dominant eigenvalues and $\phi^{\mathrm{e}}(\mathbf{x}, t)$ the dominant eigenfunctions of the time-evolving error covariance; and, $\zeta_{i}(\theta)$ a vector of random functions. The order of the truncation $p$ is also a function of time. Eq. (27) is an extension of a first order generalized polynomial chaos (GPC) expansion [81]. The ori- 

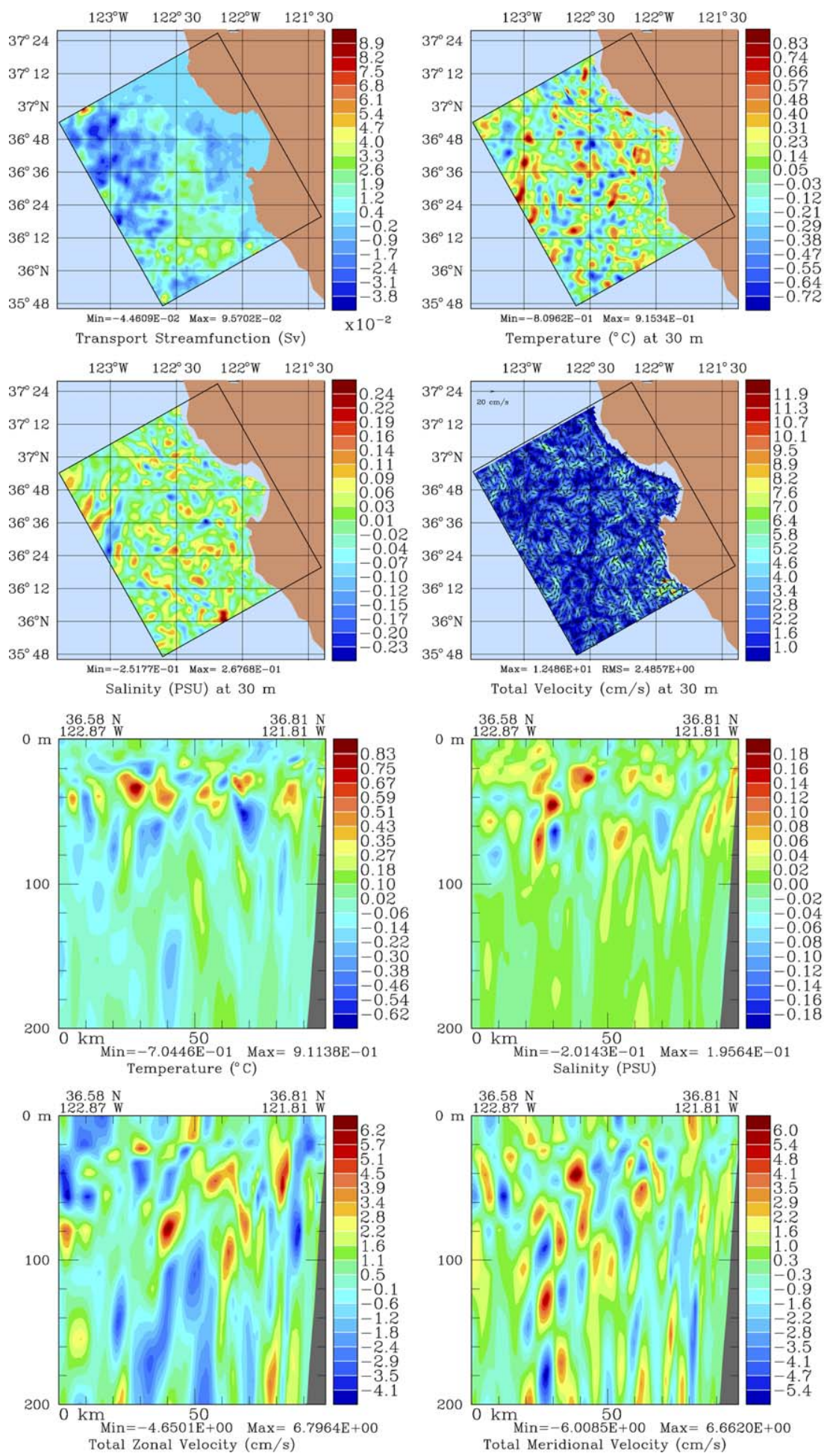

Fig. 10. Differences between a deterministic and stochastic PE simulation, after 1-day of integration. It illustrates effects of the stochastic forcings, of: amplitudes set to $\epsilon \times \|$ geostrophy $(z) \|, 1 / 2$ day decorrelation time and 1-to-2 grid point correlation in space. Shown are differences in horizontal maps of $\psi, T, S$ and $\mathbf{u}_{h}$ and in cross-sections (from offshore to the coast in Monterey Bay) of $T, S, u$ and $v$. 

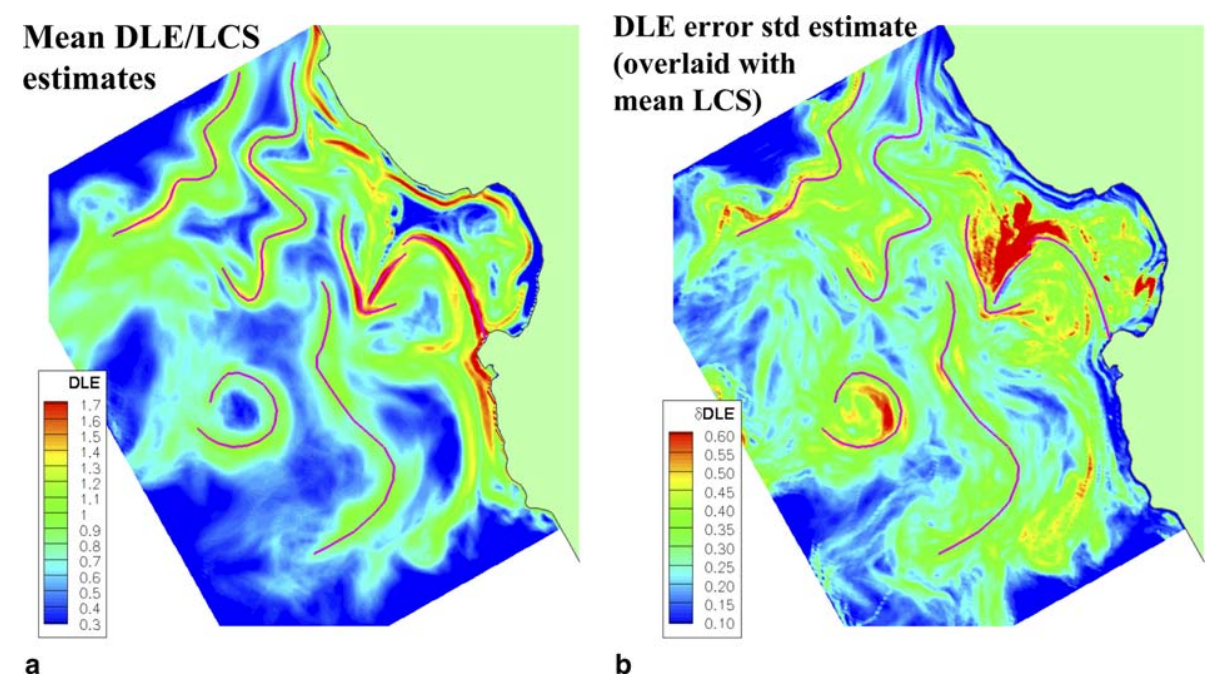

Fig. 11. ESSE uncertainty estimates for direct Lyapounov exponent fields $\left(\right.$ day $\left.^{-1}\right)$ and the corresponding Lagrangian coherent structure estimates.

ginal polynomial chaos formulation was proposed by Wiener [78]; see also [23]. It employs Hermite polynomials in terms of Gaussian random variables as the trial basis to represent stochastic processes. The GPC [81] employs more types of orthogonal polynomials and can deal with non-Gaussian random inputs more efficiently. For example, an orthogonal Karhunen-Loeve basis is used in [24] to define a PC expansion and propagate uncertainty in structural mechanics systems using a stochastic finite element approach. The coefficients of the expansion are evaluated as generalized Fourier coefficients via a Galerkin procedure. ESSE does not yet provide approximate governing equations for the bases $\phi^{\mathrm{e}}(\mathbf{x}, t)$ (Eq. (27)). It is carried out by an ensemble integration in time and importantly with data assimilation updates. Deriving such equations is an area of future research.

Other schemes that could become useful in realistic ocean DA are based on sequential Monte-Carlo particle methods [18], many of which differ as a function of the resampling strategy, e.g., genetic resampling or minimum variance branching, see $[13,17]$. Other methodologies are based on moment approximations or finitedimensional projections of densities onto manifolds (e.g. $[5,6])$.

An essential task in ocean science is the evaluation of models and their improvements. A framework for assessing uncertainties in predictions that arise from uncertainties in underlying models is presented in [33], focusing mostly on model parameters. The approach can be extended to the estimation of parameterizations and model structures [16,56]. Several of these schemes are based on Markov Chain Monte Carlo (MCMC) techniques and/or expectation-minimization algorithms. Their implementations in realistic ocean models will likely require reductions analogous to those employed in ESSE.

\section{Conclusions}

A mathematical and computational approach for comprehensive, data-driven quantifications, estimations and predictions of uncertainties for interdisciplinary ocean dynamics was developed and exemplified. The computational methodology and numerical system, error subspace statistical estimation, is based on a reduction of the uncertainties to their largest components and on an approximation to nonlinear minimum error variance estimation. Oceanic concepts were reviewed and classic equations governing the evolution of uncertainties in the Bayesian sense summarized. Novel stochastic forcing formulations for complex ocean models were introduced and a stochastic-deterministic primitive-equation-based ocean model was presented and utilized. ESSE capabilities were illustrated in three interdisciplinary data-assimilative applications. The estimation of uncertainties for physical-biogeochemical fields was carried out in Massachusetts Bay. The transfer 
549 of physical to acoustical uncertainties was exemplified in the Middle Atlantic Bight, across the shelfbreak fron-

550

551

552

553

554

555

556

557

558

559

560

561

562

563

564

565

566

567

568

569

570

571

572

573

574

575

576

577

578

579

580

581

582

583

584

585

586

587

588

589

590

591

594

tal system. Real-time stochastic ensemble predictions and assimilation of a wide range of data types were illustrated for the Monterey Bay region.

The representation, attribution and propagation of errors for four-dimensional interdisciplinary ocean estimates will require increased theoretical and applied research efforts in the coming years. The complexity of oceanic processes, from turbulent flows to climate dynamics, presents many interesting and challenging issues in uncertainty modeling. A new era of fully interdisciplinary ocean system science, quantitatively combining models and data, is emerging. It will require accurate uncertainty estimates for both measurements and dynamical models. Such estimates are also needed for novel but essential quantitative data assimilation feedbacks: adaptive sampling, adaptive model identification and adaptive model reductions and simplifications.

Validating uncertainty predictions requires a systematic comparison with observations and involves statistical studies of data-model misfits. New comprehensive ocean data sets are needed to do so. Currently, classic tools such as the root-mean-square of data-model differences and data-model pattern correlation coefficients are commonly utilized [49]. More advanced statistical schemes [38] are also needed. A variety of uncertainty metrics need to be explored and defined for specific purposes. Automated schemes for field and uncertainty evaluations should be developed by ocean and computational scientists. The derivation and utilization of emerging stochastic ocean models is another important topic. Their statistics should be fit and validated based on processes un-resolved by the deterministic models.

Relationships with other modern uncertainty quantification schemes were discussed. A promising research direction involves combinations of ESSE ideas with generalized polynomial chaos approaches, aiming for new efficient methods for adaptive multiscale error predictions. Another research area directly arises from the ESSE scheme which learns errors from data misfits. By extension, uncertainties could thus be represented as the sum of the: (i) field, (ii) its uncertainty, and (iii) the uncertainty on its uncertainty. Fuzzy information theories and imprecise probability theories [76] can then be used.

\section{Acknowledgements}

I thank Prof. G.E. Karnadiakis and Prof. J. Glimm for the invitation to prepare a manuscript for this special issue of the Journal of Computational Physics on Uncertainty Quantification. I am thankful to P.J. Haley, W.G. Leslie, A.R. Robinson, P. Abbot, C.-S. Chiu, B. Miller, J.J. McCarthy, N. Patrikalakis, H. Schmidt, A. Pang and F. Lekien for discussions and collaborations. I also thank my AOSN-II colleagues. I am grateful to the reviewers for useful comments. This work was funded under the support of the Office of Naval Research under Grant N00014-05-1-0335, N00014-01-1-0771, N00014-05-1-0370, N00014-04-10534 and PLUSNet.

\section{Appendix A. Stochastic-deterministic interdisciplinary ocean models}

\section{A.1. Deterministic coupled models}

Ocean physics model. The physical variables are temperature $T$, salinity $S$, velocity vector $\mathbf{u}=\left(\mathbf{u}_{h}, w\right)$ and pressure $p_{w}$. For this study, their mesoscale evolution is computed by the PE model (Eqs. (A.1-7), see also Section 2) of the Harvard ocean prediction system, e.g.[30]. In Eqs. (A.1-7), $(x, y, z, t=\mathbf{r}, z, t)$ is the position vector and time, $\rho_{0}$ the density of a state of reference, $g$ gravity, $f$ the vertical Coriolis frequency, $A_{\mathrm{v}}$ and $K_{\mathrm{v}}$ vertical eddy viscosities and diffusivities, and $A_{\mathrm{h}}$ and $K_{\mathrm{h}}$ their horizontal counterpart (modeled by a scaledependent filter). Atmospheric fluxes from external atmospheric models are imposed at the surface (sometimes with a flux correction). Model parameters and boundary conditions are calibrated based on ocean data and sensitivity studies. The possible outputs consist of all state variables below and of a wide range of diagnostic variables and parameters.

$$
\text { Horiz. Mom.: } \frac{\mathrm{D} \mathbf{u}_{h}}{\mathrm{D} t}+f \mathbf{e}_{3} \wedge \mathbf{u}_{\mathrm{h}}=-\frac{1}{\rho_{0}} \nabla_{\mathrm{h}} p_{w}+\nabla_{\mathrm{h}} \cdot\left(A_{\mathrm{h}} \nabla_{\mathrm{h}} \mathbf{u}_{\mathrm{h}}\right)+\frac{\partial A_{\mathrm{v}} \partial \mathbf{u}_{\mathrm{h}} / \partial z}{\partial z}
$$


Vert. Mom.: $\rho g+\frac{\partial p_{w}}{\partial z}=0$

Thermal energy: $\frac{\mathrm{D} T}{\mathrm{D} t}=\nabla_{\mathrm{h}} \cdot\left(K_{\mathrm{h}} \nabla_{\mathrm{h}} T\right)+\frac{\partial K_{\mathrm{v}} \partial T / \partial z}{\partial z}$

Cons. of salt: $\frac{\mathrm{D} S}{\mathrm{D} t}=\nabla_{\mathrm{h}} \cdot\left(K_{\mathrm{h}} \nabla_{\mathrm{h}} S\right)+\frac{\partial K_{\mathrm{v}} \partial S / \partial z}{\partial z}$

Cons. of mass: $\nabla \cdot \mathbf{u}=0$

Eqn. of state: $\rho(\mathbf{r}, z, t)=\rho\left(T, S, p_{w}\right)$

Sound speed eqn.: $c(\mathbf{r}, z, t)=C\left(T, S, p_{w}\right)$

Acoustic model. For each acoustic frequency $f$, the acoustic coupled normal mode model [7-9] solves a linearized wave equation (Eq. (A.9)) governing sound pressure $p_{\mathrm{s}}$ whose water-column parameter is the 4D sound-speed field $c$ (Eq. (A.8)). After Fourier transform over time $t$, the acoustic pressure $P_{\mathrm{s}}(\mathbf{r}, z ; f)$ (Eqs. (A.10) and (A.11)) is decomposed in the frequency domain into slowly-varying complex envelopes that modulate (mode by mode) analytic, rapidly-varying, adiabatic-mode solutions. Given sound speed, density, attenuation rate and bathymetry vertical cross-sections, the acoustic state is obtained by integrating differential equations governing the complex modal envelopes (Eqs. (A.12) and (A.13)). Model output contains sound pressure, transmission loss, and travel time, phase and amplitude of the individual modes

$$
\begin{aligned}
& \text { Wave eqn.: } \rho c^{2}(\mathbf{r}, z, t) \nabla \cdot\left(\frac{1}{\rho} \nabla p_{\mathrm{s}}(\mathbf{r}, z, t)\right)=\frac{\partial^{2} p_{\mathrm{s}}(\mathbf{r}, z, t)}{\partial t^{2}} \\
& \text { Pres.:transfer fct.: } \nabla^{2} P_{\mathrm{s}}-\frac{1}{\rho} \nabla \rho \cdot \nabla P_{\mathrm{s}}+k^{2} P_{\mathrm{s}}=-2 \frac{r_{0}}{r} \delta\left(r-r_{0}\right)\left(z-z_{0}\right) \\
& \qquad \text { where } k \doteq 2 \pi f / c(\mathbf{r}, z, t) \\
& \text { Coupledwith } P_{s}(r, z ; f) \doteq \sum_{n} \frac{r_{0}}{\sqrt{r}} P_{n}(r ; f) Z_{n}(z ; r, f) \\
& \text { Normal-modes: }\left\{\frac{\partial^{2}}{\partial z^{2}}-\frac{1}{\rho(r, z)} \frac{\partial \rho(r, z)}{\partial z} \frac{\partial}{\partial z}+\left(k(r, z)^{2}-k_{n}(r ; f)^{2}\right)\right\} \times Z_{n}(z ; r, f)=0 \\
& \text { Modal amplit.: }\left(\frac{\mathrm{d}^{2}}{\mathrm{~d} r^{2}}+k_{n}^{2}\right) P_{n}=-\sum_{m}\left(\gamma_{m n} \frac{\mathrm{d}}{\mathrm{d} r}+C_{m n}\right) P_{m}
\end{aligned}
$$

Biogeochemical model. The model used here is based on generic advection-diffusion-reaction (ADR) equations. Its state variables $\phi_{i}(x, y, z, t)$ (also called compartments or components by some biological modelers) are governed by

$$
\frac{\partial \phi_{i}}{\partial t}+\mathbf{u} \cdot \nabla \phi_{i}-\nabla_{h}\left(A_{i} \nabla_{h} \phi_{i}\right)-\frac{\partial K_{i} \partial \phi_{i} / \partial z}{\partial z}=\mathscr{B}_{i}\left(\phi_{1}, \ldots, \phi_{i}, \ldots, \phi_{7}\right)
$$

and coupled to the velocity $\mathbf{u}$ by advection. In Eqs. (A.14-20), the first term is the local time change at a point, the second is advection, and the third and fourth are diffusions. The $K_{i}^{\prime}$ 's are eddy diffusivities and the $\mathscr{B}_{i}$ 's on the right are the biological dynamics or reactions which represents all sources and sinks of $\phi_{i}$ due to, e.g., reproduction, life-stage transitions, natural mortality, predation, chemical reactions and behavior. Universal formulations for all processes inherent in the $\mathscr{B}_{i}$ 's do not yet exist and require substantial research, but most $\mathscr{B}_{i}$ 's are known to be strongly nonlinear. The $\phi_{i}$ 's chosen here for the Massachusetts Bay uncertainty prediction are: phytoplankton $P\left(\mathrm{P}_{\mathrm{NO}_{3}}\right.$ and $\left.\mathrm{P}_{\mathrm{NH}_{4}}\right)$, zooplankton $Z$, detritus $D$, nitrate $\mathrm{NO}_{3}$, ammonium $\mathrm{NH}_{4}$ and chlorophyll- $a$ Chl (see [4] for more details). Note that in Section 2, the generic notation corresponding to Eqs. (A.14-20) is used for all state variables.

\section{A.2. Stochastic models}

With model errors, a stochastic extension in Eqs. (A.1-20) is solved. Presently, physical model errors are employed: they aim to represent uncertainties due processes (e.g., sub-mesoscales, internal tides) not resolved 
629

630

631

632

633

634

636

637

638

639

640

641

642

643

644

645

646

647

649

650

651

652

653

654

655

656

657

658

659

660

661

in the deterministic PEs (Eqs. A.1-7). Using Eqs. (5) and (6) of Section 3.1.1, spatially discrete stochastic forcings are added, only in the equations which are prognostic.

(a) Time-correlations. Considering Eq. (6), uncertainties are assumed to be stationary first-order GaussMarkov processes in time, for all state variables. This is a zero-mean random process exponentially decorrelated in time. It is generated by passing white noise through a simple feedback equation. For a scalar $\tilde{w}(t)$, its sample path and variance equations are,

$$
\begin{aligned}
& \mathrm{d} \tilde{w}+\beta \tilde{w} \mathrm{~d} t=\mathrm{d} w \\
& \dot{p}_{\tilde{w}}=-2 \beta p_{\tilde{w}}+q
\end{aligned}
$$

where $\frac{1}{\beta}$ is the autocorrelation time and $w \sim(0, q)$ a white Gaussian noise in time. Setting $\dot{p}_{\tilde{w}}$ to zero at all times yields $p_{\tilde{w}}(0)=\sigma^{2}=\frac{q}{2 \beta}$. To obtain a process $\tilde{w}$ of fixed fluctuation amplitude $\sigma$ and autocorrelation time $\frac{1}{\beta}$, the variance of $w$ is set to be a constant $q=2 \beta \sigma^{2}$.

(b) $3 d$ Spatial covariances. In space, the stochastic forcing is set to be of vertically-varying amplitude. It is obtained from a white noise on a two-grid point sub-sampled grid in all 3D directions. The white noise on the coarser grid is mapped onto the actual finer grid by linear extrapolation, leading to an approximate two-grid point correlated, 3D piecewise linear white noise. For each prognostic equation, the noise variance at a given level is chosen equal to a small $\epsilon$ fraction of the amplitude of the terms involved in the dominant PE balance at that level, as determined from historical/future data variability, equations scaling at $t_{0}$ and dynamical model runs. In continuous-time, $3 \mathrm{~d}$ discrete-space, this is summarized by

$$
\begin{aligned}
& \mathrm{d} \mathbf{x}=\mathscr{M}(\mathbf{x}, t) \mathrm{d} t+\mathbf{B}^{f_{c}}(t) \mathrm{d} \tilde{\mathbf{w}}^{c} \\
& \mathrm{~d} \tilde{\mathbf{w}}^{c}=-\boldsymbol{\beta}^{c} \tilde{\mathbf{w}}^{c} \mathrm{~d} t+\mathrm{d} \mathbf{w}^{c},
\end{aligned}
$$

where $\mathbf{x} \in \mathrm{R}^{n}$ is the discrete-space PE state vector; $\mathbf{w}_{k}^{c}$ the multivariate coarse $3 \mathrm{~d}$ white noise; $\tilde{\mathbf{w}}_{k}^{c}$ the corresponding coarse $3 \mathrm{~d}$ Gauss-Markov process; $\mathscr{M}(\cdot, t)$ the PE dynamical model operator and $\mathbf{B}^{f c}(t)$ the linear extrapolation operator, from coarse to fine state.

(c) Stochastic primitive equation model. The state variables of the discrete PE model [30,11] are $\mathbf{x}=(\hat{\mathbf{u}}, \hat{\mathbf{v}}, \mathbf{T}, \mathbf{S}, \mathbf{p})^{\mathrm{T}}$. The vectors: $\hat{\mathbf{u}}$ and $\hat{\mathbf{v}}$ are internal baroclinic horizontal zonal and meridional velocities, $\mathbf{T}$ temperatures, $\mathbf{S}$ salinities and $\mathbf{p}$ (discretized $\psi$ ) barotropic stream functions. The vector components of $\mathbf{w}^{c}$ are thus $\left(\mathbf{w}_{\hat{u}}^{c}, \mathbf{w}_{\hat{v}}^{c}, \mathbf{w}_{T}^{c}, \mathbf{w}_{S}^{c}, \mathbf{w}_{\psi}^{c}\right)^{\mathrm{T}}$. Analogous definitions hold for $\tilde{\mathbf{w}}^{c}$ and its diagonal matrices of time-decorrelations $\boldsymbol{\beta}^{c}$ and noise variances $\boldsymbol{\Sigma}_{\tilde{w}}$.

The diagonal sub-matrices of time-decorrelations, $\left(\boldsymbol{\beta}_{u}, \boldsymbol{\beta}_{v}, \boldsymbol{\beta}_{T}, \boldsymbol{\beta}_{S}, \boldsymbol{\beta}_{\psi}\right)$, in general functions of position $(x, y, z)$, were here chosen $\boldsymbol{\beta}_{X}=\beta \mathbf{I}$. The decorrelation time was assumed homogeneous in space and across state-variables. The diagonal sub-matrices of noise variances $\boldsymbol{\Sigma}_{u}, \boldsymbol{\Sigma}_{v}, \boldsymbol{\Sigma}_{T}, \boldsymbol{\Sigma}_{S}$ and $\boldsymbol{\Sigma}_{\psi}$ are functions of $z$ only. Their amplitudes were set to " $\epsilon^{*}$ geostrophy", which implies:

$$
\begin{aligned}
& \boldsymbol{\Sigma}_{u}=\boldsymbol{\Sigma}_{v}=\sigma^{2}(z) \mathbf{I} \text { with } \sigma_{U}(z)=\epsilon_{U} f_{c} U(z) \\
& \boldsymbol{\Sigma}_{T}=\sigma_{T}^{2}(z) \mathbf{I} \quad \text { with } \sigma_{T}(z)=\epsilon_{T} U(z) \frac{\Delta T(z)}{L(z)} \\
& \boldsymbol{\Sigma}_{S}=\sigma_{S}^{2}(z) \mathbf{I} \quad \text { with } \sigma_{S}(z)=\epsilon_{S} U(z) \frac{\Delta S(z)}{L(z)} \\
& \boldsymbol{\Sigma}_{\psi}=\sigma_{\psi}^{2}(z) \mathbf{I} \quad \text { with } \sigma_{\psi}(z)=\epsilon_{\psi} \frac{\bar{\omega} L(z)}{U(z)}
\end{aligned}
$$

where $f_{c}$ is the central Coriolis frequency. The values of the vertically-varying characteristic scales, $U(z), \Delta T(z)$, $\Delta S(z), L(z)$ and $\bar{\omega}$, and corresponding $\epsilon$ 's are estimated from a combination of observations and dynamical model runs.

Introducing all of these terms into the PE equations leads the stochastic PE model used in the present applications: 
Inter. Barocl.: $\mathrm{d} \hat{\mathbf{u}}=\mathrm{d} \mathbf{u}^{\prime}-\mathrm{d} \overline{\mathbf{u}}^{\prime}$

Zonal Mode: $\mathrm{d} \mathbf{u}^{\prime}=\left(-\boldsymbol{\Gamma}(\mathbf{u})+\mathbf{f v}-\frac{\mathbf{g}}{\boldsymbol{\rho}_{0}} \int_{\mathbf{z}}^{0} \boldsymbol{\rho}_{\mathbf{x}} \mathrm{d} \mathbf{z}+\mathbf{F}_{u}+\mathbf{A}_{v} \mathbf{u}_{\mathbf{z z}}\right) \mathrm{d} t+\mathbf{B}_{u}^{f c} \mathrm{~d} \tilde{\mathbf{w}}_{u}^{c}$

$$
\begin{aligned}
& \text { with } \mathbf{u}=\hat{\mathbf{u}}-\frac{\mathbf{1}}{\mathbf{H}} \psi_{\mathbf{y}} . \\
& \mathrm{d} \tilde{\mathbf{w}}_{u}^{c}=-\boldsymbol{\beta}_{u} \tilde{\mathbf{w}}_{u}^{c} \mathrm{~d} t+\mathrm{d} \mathbf{w}_{u}^{c} \\
& \text { with } \tilde{\mathbf{w}}_{u}^{c}(0) \sim\left(\mathbf{0}, \boldsymbol{\Sigma}_{u}\right) \text { and } \mathbf{w}_{u}^{c} \sim\left(\mathbf{0}, 2 \boldsymbol{\beta}_{u} \boldsymbol{\Sigma}_{u}\right)
\end{aligned}
$$

Inter. Barocl.: $\mathrm{d} \hat{\mathbf{v}}=\mathrm{d} \mathbf{v}^{\prime}-\mathrm{d} \overline{\mathbf{v}}^{\prime}$

Merid. Mode: $\mathrm{d} \mathbf{v}_{t}^{\prime}=\left(-\boldsymbol{\Gamma}(\mathbf{v})-\mathbf{f u}-\frac{\mathbf{g}}{\boldsymbol{\rho}_{0}} \int_{\mathbf{z}}^{0} \boldsymbol{\rho}_{y} d \mathbf{z}+\mathbf{F}_{v}+\mathbf{A}_{v} \mathbf{v}_{\mathbf{z z}}\right) \mathrm{d} t+\mathbf{B}_{v}^{f c} \mathrm{~d} \tilde{\mathbf{w}}_{v}^{c}$

$$
\begin{aligned}
& \text { with } \mathbf{v}=\hat{\mathbf{v}}+\frac{\mathbf{1}}{H} \boldsymbol{\psi}_{\mathbf{x}} . \\
& \mathrm{d} \tilde{\mathbf{w}}_{v}^{c}=-\boldsymbol{\beta}_{v} \tilde{\mathbf{w}}_{v}^{c} \mathrm{~d} t+\mathrm{d} \mathbf{w}_{v}^{c} \\
& \text { with } \tilde{\mathbf{w}}_{v}^{c}(0) \sim\left(\mathbf{0}, \boldsymbol{\Sigma}_{v}\right) \text { and } \mathbf{w}_{v}^{c} \sim\left(\mathbf{0}, 2 \boldsymbol{\beta}_{v} \boldsymbol{\Sigma}_{v}\right) .
\end{aligned}
$$

Thermal en.: $\mathrm{d} \mathbf{T}=\left(-\boldsymbol{\Gamma}(\mathbf{T})+\mathbf{F}_{T}+\mathbf{K}_{v} \mathbf{T}_{\mathbf{z z}}\right) \mathrm{d} t+\mathbf{B}_{T}^{f c} \mathrm{~d} \tilde{\mathbf{w}}_{T}^{c}$

Balance: $\mathrm{d} \tilde{\mathbf{w}}_{T}^{c}=-\boldsymbol{\beta}_{T} \tilde{\mathbf{w}}_{T}^{c} \mathrm{~d} t+\mathrm{d} \mathbf{w}_{T}^{c}$

$$
\text { with } \tilde{\mathbf{w}}_{T}^{c}(0) \sim\left(\mathbf{0}, \boldsymbol{\Sigma}_{T}\right) \text { and } \mathbf{w}_{T}^{c} \sim\left(\mathbf{0}, 2 \boldsymbol{\beta}_{T} \boldsymbol{\Sigma}_{T}\right) \text {. }
$$

Conser. of Salt: $\mathrm{d} \mathbf{S}=\left(-\boldsymbol{\Gamma}(\mathbf{S})+\mathbf{F}_{S}+\mathbf{K}_{v} \mathbf{S}_{\mathbf{z z}}\right) \mathrm{d} t+\mathbf{B}_{S}^{f c} \mathrm{~d} \tilde{\mathbf{w}}_{S}^{c}$

$$
\begin{aligned}
& \mathrm{d} \tilde{\mathbf{w}}_{S}^{c}=-\boldsymbol{\beta}_{S} \tilde{\mathbf{w}}_{S}^{c} \mathrm{~d} t+\mathrm{d} \mathbf{w}_{S}^{c} \\
& \tilde{\mathbf{w}}_{S}^{c}(0) \sim\left(\mathbf{0}, \boldsymbol{\Sigma}_{S}\right) \quad \text { and } \mathbf{w}_{\psi}^{c} \sim\left(\mathbf{0}, 2 \boldsymbol{\beta}_{\psi} \boldsymbol{\Sigma}_{S}\right) .
\end{aligned}
$$

Barotr. Str.: $\nabla_{h} \wedge\left[\mathbf{H}^{-1} \nabla_{\mathbf{h}} \wedge \mathrm{d} \boldsymbol{\psi} \mathbf{e}_{3}\right]=-\nabla_{h} \wedge \mathrm{d} \overline{\mathbf{u}}+\mathbf{B}_{\psi}^{f c} \mathrm{~d} \tilde{\mathbf{w}}_{\psi}^{c}$

Function: $\mathrm{d} \tilde{\mathbf{w}}_{\psi}^{c}=-\boldsymbol{\beta}_{\psi} \tilde{\mathbf{w}}_{\psi}^{c} \mathrm{~d} t+\mathrm{d} \mathbf{w}_{\psi}^{c}$

$$
\text { with } \tilde{\mathbf{w}}_{\psi}^{c}(0) \sim\left(\mathbf{0}, \boldsymbol{\Sigma}_{\psi}\right) \text { and } \mathbf{w}_{\psi}^{c} \sim\left(\mathbf{0}, 2 \boldsymbol{\beta}_{\psi} \boldsymbol{\Sigma}_{\psi}\right) \text {. }
$$

671 For their numerical solutions, we refer to the reference list and to [47].

\section{References}

[1] Autonomous Ocean Sampling Network-II (AOSN-II) field exercise, August 2003. Available from: <www.mbari.org/aosn>.

[2] J.L. Anderson, A method for producing and evaluating probabilistic forecasts from ensemble model integrations, J. Climate 9 (1996) $1518-1530$.

[3] A.F. Bennett, Inverse Methods in Physical Oceanography, Cambridge Monographs on Mechanics and Applied Mathematics, Cambridge University Press, 1992.

[4] S.T. Besiktepe, P.F.J. Lermusiaux, A.R. Robinson, Coupled physical and biogeochemical data driven simulations of Massachusetts Bay in late summer: real-time and post-cruise data assimilation, Special issue on "The use of data assimilation in coupled hydrodynamic, ecological and bio-geo-chemical models of the oceans”, M. Gregoire, P. Brasseur, P.F.J. Lermusiaux (Guest Eds.), J. Mar. Sys. 40, 2003, 171-212.

[5] D. Brigo, B. Hanzon, F. LeGland, A differential geometric approach to nonlinear filtering: the projection filter, IEEE Trans. Automat. Control 43 (2) (1998) 247-252.

[6] S. Challa, Y. Bar-Shalom, V. Krishnamurthy, Nonlinear filtering via generalized Edgworth series and Gauss-Hermite quadrature, IEEE Trans. Signal Process. 48 (6) (2000) 1816-1820.

[7] C.-S. Chiu, Downslope modal energy conversion, J. Ac. Soc. Am. 95 (3) (1994) 1654-1657.

[8] C.-S. Chiu, J.H. Miller, W.W. Denner, J.F. Lynch, A three-dimensional, broadband, coupled normal-mode sound propagation modeling approach, in: O. Diachok, A. Caiti, P. Gerstoft, H. Schmidt (Eds.), Full Field Inversion Methods in Ocean and Seismic Acoustics, Kluwer Academic Publishers, Dordechet, 1995, pp. 57-62.

[9] C.-S. Chiu, J.H. Miller, J.F. Lynch, Forward coupled-mode propagation modeling for coastal acoustic tomography, J. Acoust. Soc. Am. 99 (2) (1996) 793-802.

[10] S.E. Cohn, Dynamics of short-term univariate forecast error covariances, Monthly Weather Rev. 121 (11) (1993) $3123-3149$.

[11] M.D. Cox, A primitive equation, 3-dimensional model of the ocean, Technical Report, Geophysical Fluid Dynamical Laboratory/ NOAA, Princeton, 1984. 
695

[12] D.P. Dee, On-line estimation of error covariance parameters for atmospheric data assimilation, Monthly Weather Rev. 123 (1995) 1128-1145.

[13] P. Del Moral, L. Miclo, Branching and interacting particle systems approximations of Feynman-Kac formulae with applications to non-linear filtering, in: Sminaire de Probabilits, XXXIVLecture Notes in Math., vol. 1145, Springer, Berlin, 2000 , p. 1729.

[14] T. Dickey, Emerging ocean observations for interdisciplinary data assimilation systems, J. Mar. Syst. 40-41 (2003) 5-48.

[15] S. Djurcilov, K. Kim, P.F.J. Lermusiaux, A. Pang, Visualizing scalar volumetric data with uncertainty, Comput. Graphics 26 (2) (2002) 239-248.

[16] R.O. Duda, P.E. Hart, D.G. Stork, Pattern Classification, second ed., Wiley-Interscience, New York, 2000.

[17] A. Doucet, N. De Freitas, N. Gordon (Eds.), Sequential Monte Carlo Methods in Practice, Springer, Berlin, 2001.

[18] M. Dowd, A sequential Monte Carlo approach for marine ecological prediction, Environmetrics, in press.

[19] M. Ehrendorfer, Predicting the uncertainty of numerical weather forecasts: a review, Meteorol. Zeitschrift 6 (4) (1997) $147-183$.

[20] C. Evangelinos, P.F.J. Lermusiaux, S. Geiger, R.C. Chang, N.M. Patrikalakis, Web-enabled configuration and control of legacy codes: an application to ocean modeling, Ocean Modeling, in press.

[21] G. Evensen, Inverse methods and data assimilation in nonlinear ocean models, Physica D 77 (1994) $108-129$.

[22] B.F. Farrell, P.J. Ioannou, Variance maintained by stochastic forcing of non-normal dynamical systems associated with linearly stable shear flows, Phys. Rev. Lett. 72 (8) (1994) 1188-1191.

[23] R.G. Ghanem, P. Spanos, Stochastic Finite Elements: A Spectral Approach, Springer-Verlag, New York, 1991; Physica D 133 (1999) 137-144.

[24] R. Ghanem, J. Red-Horse, Propagation of probabilistic uncertainty in complex physical systems using a stochastic finite element approach, Physica D 133 (1999) 137-144.

[25] T.C. Gard, Introduction to Stochastic Differential Equations, Marcel Dekker, New York, 1988, 234pp.

[26] C.W. Gardiner, Handbook of Stochastic Methods for Physics, Chemistry and the Natural Sciences, Springer-Verlag, 1983, 442p.

[27] A. Gelb (Ed.), Applied Optimal Estimation, MIT Press, Cambridge, MA, 1974.

[28] J. Glimm, D.H. Sharp, Prediction and the quantification of uncertainty, Physica D 133 (1999) 152-170.

[29] G. Griffiths, S. Felding, H.S.J. Roe, Biological-physical-acoustical interactions, in: A.R. Robinson, J.J. McCarthy, B.J. Rothschild (Eds.), Biological-Physical Interactions in the Ocean, The Sea, vol. 12, Wiley, New York, 2002.

[30] P.J. Haley, P.F.J. Lermusiaux, W.G. Leslie, A.R. Robinson, Harvard Ocean Prediction System (HOPS). Available from: <http:// oceans.deas.harvard.edu/HOPS/HOPS.html>.

[31] G. Haller, Lagrangian coherent structures from approximate velocity data, Phys. Fluids 14 (2002) 1851-1861.

[32] T.M. Hamill, Interpretation of rank histograms for verifying ensemble forecasts, Monthly Weather Rev. 129 (2001) $550-560$.

[33] K.M. Hanson, A framework for assessing uncertainties in simulation predictions, Physica D 133 (1999) $179-188$.

[34] E.E. Hofmann, M.A.M. Friedrichs, Predictive modeling for marine ecosystems, in: A.R. Robinson, J.J. McCarthy, B.J. Rothschild (Eds.), The Sea: Biological-Physical Interactions in the Sea, vol. 12, Wiley, New York, 2002, pp. 537-565.

[35] Holmes, P., Lumley, J.L. and G. Berkooz, Turbulence, coherent structures, dynamical systems and symmetry. First paperback edition, Cambridge Monographs on Mechanics, 1998, 420pp.

[36] K. Ide, P. Courtier, M. Ghil, A.C. Lorenc, Unified notation for data assimilation: operational, sequential and variational, J. Meteorol. Soc. Jpn. 75 (1B) (1997) 181-189.

[37] A.H. Jazwinski, Stochastic Processes and Filtering Theory, Academic Press, New York, 1970.

[38] I. Jolliffe, D. Stephenson (Eds.), Forecast Verification: A Practitioner's Guide in Atmospheric Science, Wiley, New York, 2003, p. 248.

[39] I.T. Jolliffe, D.B. Stephenson, Comments on discussion of verification concepts in forecast verification: A practitioner's guide in atmospheric science, Weather Forecasting 20 (2005) 796-800.

[40] E. Kalnay, Atmospheric Modeling, Data Assimilation and Predictability, Cambridge University Press, Cambridge, 2003,341 pp.

[41] G.E. Karniadakis, (Ed.), 2002. Quantifying Uncertainty in CFD. Special issue of J. Fluids Eng.

[42] R. Kleeman, Measuring dynamical prediction utility using relative entropy, J. Atmos. Sci. 59 (2002) $2057-2072$.

[43] W.A. Kuperman. Underwater Acoustics. Encyclopedia of Physical Science and Technology, Elsevier Science Ltd., pp. 317-338.

[44] H.J. Kushner, Approximations to optimal nonlinear filters, IEEE Trans. Auto. Contr. 12 (5) (1967) 546-556.

[45] C.M. Lalli, T.R. Parsons, Biological Oceanography: An Introduction, Butterworth-Heinemann, Oxford, $1997,314$.

[46] G. Kallianpur, Stochastic Filtering Theory, Applications of Mathematics, vol. 13, Springer-Verlag, New York-Berlin, 1980.

[47] P.E. Kloeden, E. Platen, Numerical solution of stochastic differential equationsApplications of Mathematics, vol. 23, Springer-Verlag, Berlin, 1992, p. 632.

[48] P.F.J. Lermusiaux, A.R. Robinson, Data assimilation via error subspace statistical estimation, Part I: theory and schemes, Monthly Weather Rev. 127 (7) (1999) 1385-1407.

[49] P.F.J. Lermusiaux, Data assimilation via error subspace statistical estimation, Part II: Middle Atlantic Bight shelfbreak front simulations and ESSE validation, Monthly Weather Rev. 127 (7) (1999) 1408-1432.

[50] P.F.J. Lermusiaux, Estimation and study of mesoscale variability in the Strait of Sicily, Dyn. Atmos. Oceans 29 (1999) $255-303$.

[51] P.F.J. Lermusiaux, D.G. Anderson, C.J. Lozano, On the mapping of multivariate geophysical fields: error and variability subspace estimates, Q.J.R. Meteorol. Soc. (April B) (2000) 1387-1430.

[52] Lermusiaux, P.F.J., Evolving the subspace of the three-dimensional multiscale ocean variability: Massachusetts Bay. J. Marine Systems, Special issue on "Three-dimensional ocean circulation: Lagrangian measurements and diagnostic analyses", 29/1-4, 2001, $385-422$. 
[53] P.F.J. Lermusiaux, A.R. Robinson, Features of dominant mesoscale variability, circulation patterns and dynamics in the Strait of Sicily, Deep Sea Res. 48 (9) (2001) 1953-1997.

[54] P.F.J. Lermusiaux, On the mapping of multivariate geophysical fields: sensitivity to size, scales and dynamics, J. Atmos. Oceanic Technol. 19 (2002) 1602-1637.

[55] P.F.J. Lermusiaux, A.R. Robinson, P.J. Haley, W.G. Leslie, Advanced interdisciplinary data assimilation: filtering and smoothing via error subspace statistical estimation, in: Proceedings of "The OCEANS 2002 MTS/IEEE” Conference, Holland Publications, 2002, pp. 795-802.

[56] P.F.J. Lermusiaux, C. Evangelinos, R. Tian, P.J. Haley, J.J. McCarthy, N.M. Patrikalakis, A.R. Robinson, H. Schmidt, Adaptive coupled physical and biogeochemical ocean predictions: a conceptual basis, in: F. Darema, (Ed.), "Computational Science - ICCS 2004", Lecture Notes in Computer Science, vol. 3038, 2004, pp. 685-692.

[57] P.F.J. Lermusiaux, F. Lekien, Dynamics and lagrangian coherent structures in the ocean and their uncertainties, in: J. Marsden, J. Scheurle (Eds.), Proceedings of "Dynamical System Methods in Fluid Dynamics", Oberwolfach, Germany, in press.

[58] J.L. Lions, Optimal Control of Systems Governed by Partial Differential Equations, Springer Verlag, Berlin, 1971.

[59] J. Lynch, A. Newhall, B. Sperry, G. Gawarkiewicz, P. Tyack, C.-S. Chiu, Spatial and temporal variations in acoustic propagation characteristics at the New England shelfbreak front, IEEE J. Oceanic Eng. 28 (2001) 129-150.

[60] N.C. Makris, P. Ratilal, D.T. Symonds, R.W. Nero, Continuous wide area monitoring of fish shoaling behavior with acoustic waveguide sensing and bioclutter implications, J. Acoust. Soc. Am. 115 (2004) 2618.

[61] P. Malanotte-Rizzoli (Ed.), Modern Approaches to Data Assimilation in Ocean Modeling, Elsevier, Amsterdam, 1996.

[62] J.J. McCarthy, A.R. Robinson, B.J. Rothschild, Biological-Physical Interactions, in: Allan R. Robinson, James J. McCarthy, Brian J. Rothschild (Eds.), The Sea: Emergent Findings and New Directions, The Sea, vol. 12, Wiley, New York, 2002 (Chapter 1).

[63] R.N. Miller, E.F. Carter, S.L. Blue, Data assimilation into nonlinear stochastic models, Tellus A 51 (1999) 167-194.

[64] R.N. Miller, L.L. Ehret, Ensemble generation for models of multimodal systems, Monthly Weather Rev. 130 (9) (2002) 2313-2333.

[65] N.M. Patrikalakis, J.J. McCarthy, A.R. Robinson, H. Schmidt, C. Evangelinos, P.J. Haley, S. Lalis, P.F.J. Lermusiaux, R. Tian, W.G. Leslie, W. Cho, Towards a dynamic data-driven system for rapid adaptive interdisciplinary ocean forecasting, in: F. Darema (Ed.), Invited paper in Dynamic Data-Driven Application Systems, Kluwer Academic Publishers, Amsterdam, 2005.

[66] J. Pedlosky, Geophysical Fluid Dynamics, second ed., Springer-Verlag, Berlin, 1987.

[67] N. Pinardi, J. Woods (Eds.), Ocean Forecasting: Conceptual Basis and Applications, Springer Verlag, Berlin, 2002, p. 472.

[68] A.R. Robinson, P.F.J. Lermusiaux, N.Q. Sloan, Data assimilation, in: The Sea: The Global Coastal Ocean, in: K.H. Brink, A.R. Robinson (Eds.), Processes and Methods, vol. 10, Wiley, NY, 1998, pp. 541-594.

[69] A.R. Robinson, J.J. McCarthy, B.J. Rothschild, Interdisciplinary ocean science is evolving and a systems approach is essential, J. Mar. Sys. 22 (1999) 231-239.

[70] A.R. Robinson, P.F.J. Lermusiaux, Data Assimilation in ModelsEncyclopedia of Ocean Sciences, Academic Press Ltd., London, 2001, pp. 623-634.

[71] A.R. Robinson, P.F.J. Lermusiaux, Data assimilation for modeling and predicting coupled physical-biological interactions in the sea, in: A.R. Robinson, J.J. McCarthy, B.J. Rothschild (Eds.), The Sea: Biological-Physical Interactions in the Sea, vol. 12, Wiley, New York, 2002, pp. 475-536.

[72] L. Sirovich, Analysis of turbulent flows by means of the empirical eigenfunctions, Fluid Dyn. Res. 8 (1991) 85-100.

[73] L.A. Smith, J.A. Hansen, Extending the limits of ensemble forecast verification with the minimum spanning tree, Monthly Weather Rev. 132 (2004) 1522-1528.

[74] D.B. Stephenson, F.J. Doblas-Reyes, Statistical methods for interpreting Monte Carlo ensemble forecasts, Tellus 52A (2000) $300-322$.

[75] Talagrand, O., R. Vautard, and B. Strauss, Evaluation of probabilistic prediction systems, in: Proceedings of ECMWF Workshop on Predictability, Shinfield Park, Reading, United Kingdom, ECMWF, 1999, pp. 1-25.

[76] P. Walley, Statistical Reasoning with Imprecise Probabilities, Monographs on Statistics and Applied Probability Series, vol. $42,1991$.

[77] A. Weisheimer, L.A. Smith, K. Judd, A new view of seasonal forecast skill: Bounding boxes from the DEMETER ensemble forecasts, Tellus 57A (2005) 265-279.

[78] N. Wiener, The homogeneous chaos, Am. J. Math. 60 (1938) 897-936.

[79] D.S. Wilks, The minimum spanning tree histogram as a verification tool for multidimensional ensemble forecasts, Monthly Weather Rev. 132 (2004) 1329-1340.

[80] C. Wunsch, The Ocean Circulation Inverse Problem, Cambridge University Press, Cambridge, 1996.

[81] D. Xiu, G.E. Karniadakis, Modeling uncertainty in flow simulations via generalized polynomial chaos, J. Comput. Phys. 187 (2003) $137-167$.

[82] M. Zakai, On the optimal filtering of diffusion processes, Zeit. Wahrsch. 11 (1969) 230-243. 